\title{
Recognition between a short unstructured peptide and a partially folded fragment leads to the thioredoxin fold sharing native-like dynamics
}

\author{
Andrés Binolfi, ${ }^{1}$ Claudio O. Fernández, ${ }^{1,2}$ Mauricio P. Sica, ${ }^{3}$ José M. Delfino, ${ }^{4 *}$ and \\ Javier Santos ${ }^{3,4 \star}$ \\ ${ }^{1}$ Instituto de Biología Molecular y Celular de Rosario, Consejo Nacional de Investigaciones Científicas y Técnicas, \\ Universidad Nacional de Rosario, Suipacha 531, S2002LRK Rosario, Argentina \\ 2 Department of NMR-based Structural Biology, Max Planck Institute for Biophysical Chemistry, Am Fassberg 11, D-37077 \\ Göttingen, Germany and DFG Research Center for the Molecular Physiology of the Brain (CMPB), Göttingen, Germany \\ ${ }^{3}$ Departamento de Ciencia y Tecnología, Universidad Nacional de Quilmes, Roque Sáenz Peña 352, B1876XD Bernal, \\ Buenos Aires, Argentina \\ ${ }^{4}$ Departmento de Química Biológica e Instituto de Química y Fisicoquímica Biológicas (IQUIFIB), Facultad de Farmacia y \\ Bioquímica, Universidad de Buenos Aires, Junín 956, C1113AAD Buenos Aires, Argentina
}

\begin{abstract}
Thioredoxins (TRXs) constitute attractive $\alpha / \beta$ scaffolds for investigating molecular recognition. The interaction between the recombinant fragment spanning the sequence 1-93 of full-length TRX (TRX1-93) and the synthetic peptide comprising residues 94-108 (TRX94-108), plus a C-terminal tyrosine tag (the numbering scheme used in entry pdb 2TRX is used throughout the article, two complementary moieties of $E$. coli TRX, brings about the consolidation of a native-like complex. Despite its reduced thermodynamic stability, this complex is able to acquire fine structural features remarkably similar to those characteristic of full-length TRX, namely, hydrodynamic behavior, assessed by diffusion-ordered spectroscopy (DOSY)-NMR; the pattern of secondary structure, as revealed by three-bond $\mathrm{HNH} \alpha$ coupling constants and secondary shifts for $\mathrm{H} \alpha / \mathrm{CO} /$ $\mathrm{C} \alpha / \mathrm{C} \beta$; native-like tertiary structural signatures revealed by near-UV circular dichroism (CD) spectroscopy. The complex exhibits a relaxation behavior compatible with that expected for a native-like structure. However, heteronuclear nuclear Overhauser effect (NOE)s reveal an enhanced dy-
\end{abstract}

namics for the complex by comparison with full-length TRX. Furthermore, higher $R_{2}$ values for residues $43-50$ and 74-89 would likely result from an exchange process modulated by the peptide at the interface region. The slow kinetics of the consolidation reaction was followed by $\mathrm{CD}$ and realtime NMR. Equilibrium titration experiments by NMR yield a $K_{\mathrm{D}}$ value of $1.4 \pm 1.0 \mu M$ and a second low-affinity ( $>150$ $\mu M)$ binding event in the vicinity of the active site. Molecular dynamics simulations of both the isolated fragment TRX1-93 and the complex suggest the destabilization of $\alpha 2$ and $\alpha 3$ helical elements and the persistence of $\beta$-structure in the absence of TRX94-108. Altogether, structural and dynamic evidence presented herein points to the key role played by the C-terminal helix in establishing the overall fold. This critical switch module endows reduced TRX with the ability to act as a cooperative folding unit.

Proteins 2012; 80:1448-1464.

(C) 2012 Wiley Periodicals, Inc.

Key words: thioredoxin; tertiary structure; NMR; protein fragment; structure consolidation; backbone dynamics; molecular dynamics simulation.

Additional Supporting Information may be found in the online version of this article.

Abbreviations: ACN, acetonitrile; CD, circular dichroism; DTT, dithiothreitol; MDS, molecular dynamics simulation; HPLC, high-performance liquid chromatography; $R_{\mathrm{h}}$, the hydrodynamic radius; SEC, size-exclusion chromatography; TRX, thioredoxin; TRX1-93, the recombinant fragment spanning the sequence 1-93 of full-length TRX; TRX94-108, the synthetic peptide comprising residues $94-108$, plus a C-terminal tyrosine tag (the numbering scheme used in entry pdb 2 TRX is used throughout the paper.

Grant sponsor: Agencia Nacional de Promoción Científica y Tecnológica (ANPCyT); Grant number: PME2003-0026; Grant sponsors: Consejo Nacional de Investigaciones Científicas y Técnicas (CONICET), Universidad de Buenos Aires (UBACyT), Universidad Nacional de Quilmes (UNQ), Instituto de Biología Molecular y Celular de Rosario (IBR-CONICET).

${ }^{*}$ Correspondence to: José M. Delfino, Departmento de Química Biológica e Instituto de Química y Fisicoquímica Biológicas (IQUIFIB), Facultad de Farmacia y Bioquímica, Universidad de Buenos Aires, Junín 956, C1113AAD Buenos Aires, Argentina. E-mail: delfino@qb.ffyb.uba.ar or Javier Santos, Department of Biological Chemistry and Institute of Biochemistry and Biophysics (IQUIFIB), School of Pharmacy and Biochemistry, University of Buenos Aires, Junín 956, C1113AAD Buenos Aires, Argentina. E-mail: jsantos@qb.ffyb.uba.ar.

Received 2 September 2011; Revised 27 December 2011; Accepted 11 January 2012

Published online 27 January 2012 in Wiley Online Library (wileyonlinelibrary.com). DOI: 10.1002/prot.24043 


\section{INTRODUCTION}

The consolidation of the tertiary structure of a protein is a crucial step in the folding reaction. This process is generally cooperative and represents the outcome of a complex interplay between local as well as long range interactions. $^{1-3}$ The formation of contact networks results most likely in the expulsion of interstitial water, maximizing compactness of the overall structure, achieving minimal frustration, ${ }^{4-7}$ and attaining global stability. The spatial and temporal coordination of these contacts may encrypt the basis of native state stability, and perhaps, mechanistic clues that may enlighten general concepts on the acquisition of protein structure.

A fascinating problem is whether after the initial collapse, ${ }^{8}$ tertiary structure results from the conformational selection of interacting folding modules, or whether it is gained along the binding process by the induced fit of the counterparts. ${ }^{9-13}$ Modern views aim at reconciling these extremes into a single integrated picture. ${ }^{14,15}$ Significant advances in this field were achieved by studying protein-ligand and protein-protein interactions. ${ }^{16-23}$ Importantly, under folding conditions, the native state would be selected from a limited collection of structural possibilities, each one corresponding to a particular hydrogen-bonded arrangement of alpha-helices and/or strands of beta-sheets. ${ }^{24}$

Rather than a single rigid structure, the new view of the folded state represents it best by an ensemble of conformations in a landscape. ${ }^{15,25,26}$ For instance, the ensemble of ubiquitin in solution samples the structural heterogeneity observed in various crystal structures of free and bound forms of this protein. ${ }^{27}$ The presence of ligands, changes in temperature or $\mathrm{pH}$, or a variation in the concentration of osmolytes or chaotropic agents shift the concentration of species in equilibrium in the folded and unfolded ensembles by altering their relative stabilities. ${ }^{28-30}$ In addition, energetic barriers among conformational states determine the kinetics of the process. The biological relevance of thermodynamic $\left(\Delta G_{\mathrm{NU}}\right)$ and kinetic $\left(\Delta G_{\mathrm{N} *}\right)$ stabilities is a key topic of central discussion nowadays in structural biology. $31-33$ Protein dynamics sheds light on areas such as signaling networks, drug design, or protein evolvability. ${ }^{34-36}$

E. coli thioredoxin (TRX), a 108 residue globular and monomeric $\alpha / \beta$ protein is an extensively studied model system. ${ }^{37}$ The structure of full-length TRX has been investigated by both $\mathrm{NMR}^{38}$ and X-ray crystallography. ${ }^{39}$ The assignments of ${ }^{15} \mathrm{~N}$ NMR spectra are available for both reduced and oxidized TRX, 40 and the backbone dynamics of both forms was also studied by NMR. ${ }^{41}$ In addition, their hydrogen-exchange behavior is also known. ${ }^{42}$ Interestingly, individual segments of TRX corresponding to the $\mathrm{N}$-terminal 73 residue fragment (TRX1-73) and the C-terminal 35 residue peptide (TRX74-108) showed the lack of native secondary or tertiary structures. However, when both partners mix, mutual interaction-characterized by a $K_{\mathrm{D}}$ value of $49 \mathrm{nM}$-leads to refolding, yielding a nativelike TRX complex (Supporting Information Table S1). 42 By the same token, fragments TRX1-37 and TRX38-108 also interact to give a complex with native properties but endowed with less stability than the pair TRX1-73/TRX74108. ${ }^{43}$ Remarkably, both complexes exhibit marginal TRX enzymatic activity against a polypeptide substrate like insulin $\left(<1 \%\right.$, Supporting Information Table S1), ${ }^{44}$ In both cases, this behavior can be attributed to the disturbance caused by the charges of the new termini due to their proximity to the active site.

Thus, TRX appears to be a very malleable system to explore the consequences of surface-surface intramolecular interactions in regard to the consolidation of tertiary structure. The astonishing plasticity of the interfaces between TRX structural elements was illustrated by high-resolution NMR using a human/E. coli chimeric molecule. ${ }^{45}$ In this protein construct, two new interfaces are created: one involving $\beta$ strands 2 and 4, and a second, linking $\alpha$ helix 2 with the C-terminal $\alpha$-helix. These elements are well packed with minimal structural perturbation. Particularly important, the hydrogen bonding net between the two strands remains intact. In addition, some favorable apolar interactions between $\alpha 2$ and C-terminal helices in the hybrid molecule are conserved as in the parental variants. The new interfaces do not exhibit increased mobility, by comparison with the main body of the protein, and the average order parameter $\left(S^{2}\right)$ value for the entire protein indicates a native-like backbone dynamics. However, the stability of the chimeric variant is greatly reduced. 46

In line with the aforementioned plasticity characteristic of E. coli TRX, we recently showed that the recombinant fragment spanning the sequence 1-93 of full-length TRX (TRX1-93) of E. coli TRX is able to interact favorably with the synthetic peptide comprising residues 94-108 (TRX94-108), plus a C-terminal tyrosine tag (the numbering scheme used in entry pdb 2TRX is used throughout the article. ${ }^{47}$ In particular, our studies concerning peptide TRX94-108 suggest that interactions of this moderately amphipathic peptide with local apolar environments-like that provided by detergent molecules or hydrophobic surfaces-are enough to stabilize a helical conformation. In other words, the acquisition of structure in this module does not require the fine structure of a template represented in this case by the counterpart fragment TRX1-93. ${ }^{48}$ However, molecular dynamics simulations (MDS) of TRX94-108 in the presence of the detergent SDS enabled us to hypothesize that stabilization of the secondary structure of this peptide may require multiple contacts between selected residues and the complementary apolar surface. Furthermore, we have recently shown that residues L99, F102, and L103 play a key role in stabilizing the $\alpha$-helical conformation of peptide TRX94-108, leading to the consolidation of the tertiary structure of the complex. ${ }^{49}$ In this context, we hypothe- 
size that folding depends on the formation of a cooperative tertiary unit based on the interaction between (helix $\alpha 5$ of peptide TRX94-108 and (helix $\alpha 3$ of fragment TRX1-93.49 Because isolated peptide TRX94-108 remains unstructured in solution, and fragment TRX1-93 exhibits features of partially folded states, folding of both peptide and fragment should occur concomitantly. Both local (intrahelical interactions) and tertiary contacts (between the C-terminal helix and the rest of the protein) should be coordinated to reach the native-like state of TRX. However, the detailed structure of this complex remains unknown. In this regard, to advance the characterization of the conformational ensemble, it is important to establish the extent of flexibility of the main chain.

In this article, we focus on the gain of structure and characteristic dynamics experienced by fragment TRX1-93 on binding the cognate peptide, taking advantage of the fine detail provided by modern NMR methods. To this end, we have completed the assignment of resonances corresponding to the backbone and studied its conformation and dynamics at the level of individual amino acids residues on the reduced form of TRX1-93 refolded in the presence of peptide TRX94-108. This knowledge sets the stage for the understanding of the mechanism leading to complex formation. The ensuing MDS analysis highlights underlying features of the partners involved in this molecular recognition process. By exploring the role of the fine interplay between moieties, we were able to comprehend key issues of the dynamics of the interaction and their implications for the attainment of the folded state of the protein.

\section{MATERIALS AND METHODS}

\section{Peptide synthesis and purification}

Peptide TRX94-108, of sequence LSKGQLKEFLDANLAY, was synthesized and partially purified by GenScript Corp (Piscataway, NJ). The purification was completed by high-performance liquid chromatography (HPLC; Rainin Dynamax, NY) using a reverse phase C18 semipreparative column equilibrated in $0.05 \%$ aqueous trifluoroacetic acid (TFA). TRX94-108 was eluted with a linear gradient from 30 to $45 \%$ aqueous acetonitrile (ACN), $0.05 \%$ TFA; the peptide typically elutes at $40 \%$ ACN. Fractions containing $>98.0 \%$ pure peptide were pooled and lyophilized. A Cterminal tyrosine tag was added to allow the measurement of concentration by absorbance in the near-UV region (using an extinction coefficient: $\varepsilon_{280 \mathrm{~nm}}=1490 M^{-1} \mathrm{~cm}^{-1}$ ). The mass of peptides was checked after HPLC by electrospray ionization mass spectrometry.

\section{Protein expression, purification and refolding}

Unlabeled TRX and TRX1-93 were expressed and purified as previously described. ${ }^{47,49}{ }^{15} \mathrm{~N}$ - and ${ }^{13} \mathrm{C} /{ }^{15} \mathrm{~N}$-la- beled proteins were prepared from E. coli cultures grown in M9 minimal medium, supplemented with ${ }^{15} \mathrm{~N}-\mathrm{NH}_{4} \mathrm{Cl}$ and ${ }^{13} \mathrm{C}$-D-glucose obtained from Cambridge Isotope Laboratories (Andover, MA). The ESI ion trap mass spectrometer (Thermo Finnigan, San José, CA) was used to confirm the expected masses of unlabeled and labeled proteins. $\mathrm{Pu}-$ rity was checked by HPLC and sodium dodecyl sulfate polyacrylamide gel electrophoresis. 50

Partial refolding of urea-unfolded fragment TRX1-93 $(100 \mu \mathrm{M})$ was performed by dialysis at $4^{\circ} \mathrm{C}$ for $16 \mathrm{~h}$. For real-time NMR measurements (see Results), and for equilibrium titration of fragment TRX1-93 with peptide TRX94-108, the former was used without subsequent concentration to avoid aggregation in along the refolding process. In the case of equilibrium titration experiments, peptide TRX94-108 was added up to 5:1 molar ratio (peptide to fragment) and incubated for $16 \mathrm{~h}$ at room temperature. To characterize the binding process, for each heteronuclear single-quantum coherence (HSQC) spectrum, cross-peak heights and peak volumes were measured as a function of peptide to fragment molar ratio for a set of well-resolved cross-peaks. The parameter $K_{\mathrm{D}}$ was individually calculated for each residue assuming a model of 1:1 peptide to fragment stoichiometry. In addition, a global $K_{\mathrm{D}}$ was fitted to the data after normalization of peak heights to the highest peptide to fragment molar ratio assayed.

For NMR assignment experiments, the dialyzed fragment was diluted to $50 \mu \mathrm{M}$ in $20 \mathrm{mM}$ TrisHCl buffer, $100 \mathrm{mM} \mathrm{NaCl}, 1.0 \mathrm{mM}$ dithiothreitol (DTT), pH 7.3. Peptide was then added at a 5:1 molar ratio, and the mixture was incubated at $20^{\circ} \mathrm{C}$ for $5 \mathrm{~h}$. Finally, the complex was concentrated up to $500 \mu \mathrm{M}$ by ultrafiltration. This protocol prevents protein aggregation and allowed us to reach a final peptide concentration of $1.5 \mathrm{mM}$.

\section{NMR spectroscopy, data acquisition, and processing}

NMR spectra were acquired at $20^{\circ} \mathrm{C}$ on a Bruker Avance II 600 spectrometer. The following three-dimensional triple-resonance experiments were collected to obtain sequence-specific assignments for the backbone of TRX1-93: CBCANH, CBCACONH, HNCO, HN(CA)CO, HNCA. HN(CO)CA, HNHA, ${ }^{15} \mathrm{~N}$ TOCSY HSQC, and ${ }^{15} \mathrm{~N}$ NOESY HSQC. Acquisition parameters for the triple resonance experiments were as follows: $\mathrm{CBCANH}$ : field $(\mathrm{MHz})$ : 600; real points: $50\left({ }^{15} \mathrm{~N}\right), 110\left({ }^{13} \mathrm{C}\right), 2048\left({ }^{1} \mathrm{H}\right)$; spectral width (ppm): $36\left({ }^{15} \mathrm{~N}\right), 75\left({ }^{13} \mathrm{C}\right), 14\left({ }^{1} \mathrm{H}\right)$. CBCACONH: 600; 50, 110, 2048; 36, 75, 14. HNCO: 600; 50, 128, 2048; 36, 15, 14. HNCACO: 600; 50, 80, 2048; 36, 15, 14. HNCA: 600; 40, 80, 2048; 36, 40, 14. $\mathrm{HN}(\mathrm{CO}) \mathrm{CA}: 600 ; 40,96,2048 ; 36,40,14$. HNHA: 600; 40, $128\left({ }^{1} \mathrm{H}\right), 2048 ; 38,14\left({ }^{1} \mathrm{H}\right), 14 .{ }^{15} \mathrm{~N}$ TOCSY HSQC: 600; 40, $64\left({ }^{1} \mathrm{H}\right), 2048 ; 38,14\left({ }^{1} \mathrm{H}\right), 14 .{ }^{15} \mathrm{~N}$ NOESY HSQC: $600 ; 40,64\left({ }^{1} \mathrm{H}\right), 2048 ; 38,14\left({ }^{1} \mathrm{H}\right), 14$. All these 
experiments were acquired at $20^{\circ} \mathrm{C}$ on a sample of fragment TRX1-93 in complex with peptide TRX94-108 prepared as described above. The backbone assignment of the complex with the unlabeled peptide listing all measured ${ }^{1} \mathrm{H} \alpha,{ }^{1} \mathrm{HN}$, ${ }^{15} \mathrm{~N},{ }^{13} \mathrm{CO},{ }^{13} \mathrm{C} \alpha$, and ${ }^{13} \mathrm{C} \beta$ chemical shifts will be deposited at the Biological Magnetic Resonance Data Bank.

Secondary shift values were calculated as the differences between the measured $\mathrm{H} \alpha / \mathrm{CO} / \mathrm{C} \alpha / \mathrm{C} \beta$ chemical shifts and the empirical random coil values reported by Wishart et al. 51,52 4,4-Dimethyl-4-silapentane-1-sulfonic acid was used for chemical shift referencing.

Three-bond $\mathrm{HNH} \alpha$ coupling constants $\left({ }^{3} \mathrm{JHNH}_{\mathrm{HN}}\right)$ were determined from the ratio between the intensities of the diagonal and cross-peak in the HNHA experiment, 53 according to the relation of intensities:

$$
-\tan ^{2}\left(2 \pi \zeta^{3} J_{\mathrm{HNH} \alpha}\right)=\frac{I_{\text {Cross-peak }}}{I_{\text {Diagonal peak }}}
$$

where $\zeta$ delay is $13.5 \mathrm{~ms}$.

For calculation of secondary coupling constants $\left(J_{\text {obs }}-\right.$ $\left.J_{\mathrm{rc}}\right)$, random coil values for each amino acid were subtracted from the experimental values. Two sets of random coil ${ }^{3} J_{\mathrm{HNH} \alpha}$ couplings were evaluated, the ones reported by Serrano, 54 and the ones reported by Smith et al. 55 For calculation of the secondary structure propensity (SSP) score, ${ }^{1} \mathrm{H} \alpha,{ }^{13} \mathrm{C} \alpha,{ }^{13} \mathrm{C} \beta,{ }^{13} \mathrm{CO}, \mathrm{HN}$, and ${ }^{15} \mathrm{~N}$ amide chemical shift values were used. ${ }^{53}$

For refolding experiments, band-selective optimizedflip-angle short-transient (SOFAST) HMQC, real-time two-dimensional (2D) NMR spectra ${ }^{56}$ were acquired every $7 \mathrm{~min}$ after addition of peptide TRX94-108 to the ${ }^{15} \mathrm{~N}$-labeled fragment TRX1-93.

Acquisition, processing, and visualization of the spectra were performed by using TOPSPIN 2.0 (Bruker), SPARKY 3 (University of California, San Francisco, CA) and CARA program (Computer-Aided Resonance Assignment, Institute for Molecular Biology and Biophysics, Zürich, Switzerland).

\section{Determination of hydrodynamic radii and compactness}

The hydrodynamic radius $\left(R_{\mathrm{h}}\right)$ of the isolated forms of peptide TRX94-108 and fragment TRX1-93, and the $R_{\mathrm{h}}$ of complex TRX94-108/TRX1-93 was measured by diffusion order spectroscopy (DOSY-NMR). In addition, we included in our $R_{\mathrm{h}}$ determinations full-length TRX as a control sample. NMR spectra were acquired on a Bruker Avance $600 \mathrm{MHz}$ spectrometer. Pulsed-field gradient NMR experiments were collected at $20^{\circ} \mathrm{C}$, using the PGSled pulse sequence. ${ }^{57}$ Samples were prepared in $\mathrm{D}_{2} \mathrm{O}$ and dioxane was added as an internal standard $\left(R_{\mathrm{h}}=\right.$ $2.12 \AA$ ) and viscosity probe. For this purpose, lyophilized peptide was directly dissolved in $20 \mathrm{mM}$ Tris HCl buffer, $100 \mathrm{mM} \mathrm{NaCl}, 1.0 \mathrm{mM}$, DTT, pH 7.3 in $\mathrm{D}_{2} \mathrm{O}$, and in the case of the fragment, the buffer was changed to the above by ultrafiltration (Amicon). The $R_{\mathrm{h}}$ of fragment, peptide, and complex was calculated using equation:

$$
R_{\mathrm{h}} \mathrm{p}=\frac{d_{\text {dioxane }}}{d_{\mathrm{p}}} \times R_{\mathrm{h}_{\text {dioxane }}}
$$

where $d_{\mathrm{p}}$ and $d_{\text {dioxane }}$ stand for the decay coefficients of the NMR signals. These values are proportional to the true diffusion coefficients of the polypeptide and dioxane, respectively. A series of 20 one-dimensional spectra was collected as a function of gradient amplitude. The gradient strength was shifted from 0.67 to $32.03 \mathrm{G} \mathrm{cm}^{-1}$ in a linear manner. ${ }^{57}$

In addition, we performed ${ }^{1} \mathrm{H}_{-}{ }^{15} \mathrm{~N}$ HET-SOFAST HMQC NMR experiments to study qualitatively the extent of compactness along the polypeptide chain of the protein species. 58 The reduction of ${ }^{1} \mathrm{H}-{ }^{1} \mathrm{H}$ spin diffusion (or $\mathrm{NOE}$ ) is measured as an increase in the parameter $\lambda_{\mathrm{NOE}}$, the ratio between the intensity of amide peaks in the presence of saturation of aliphatic ${ }^{1} \mathrm{H}$ and that in a control spectrum without saturation $\left(I_{\mathrm{sat}} / I_{\mathrm{ref}}\right)$. Thus, the analysis of this ratio provides insights into the aliphatic proton density in the surroundings of each amide group. Selective pulses and delays incorporated into the pulse sequence were identical to those reported by Schanda et al..$^{58}$ For reference, we used the empirical threshold values of $\lambda_{\mathrm{NOE}}$ measured for well-folded, molten globular and completely unfolded proteins reported by these authors.

\section{Backbone mobility}

${ }^{15} \mathrm{~N}$ relaxation data were acquired using modern versions of pulse sequences based on those described by Farrow et al. ${ }^{59-61}$ Spectra used for longitudinal relaxation $T_{1}$ $\left(1 / R_{1}\right)$ analysis were collected using the following relaxation delay times (in ms): 5, 20,80,300, 750, 1500, and 2250. $T_{2}$ data $\left(1 / R_{2}\right)$ were measured using a pulse sequence using a the Carr-Purcell-Meiboom-Gill pulse sequence (CPMG) pulse train with the following relaxation delays (in ms): 20, 350, 40, 250, 60, 200, 90, 160, and 120. Duplicate spectra were collected at several time points to estimate uncertainty. The delay between $180^{\circ}$ pulses in the CPMG pulse train was $1 \mathrm{~ms}$. To determine $T_{1}$ and $T_{2}$ relaxation rates resonance heights were extracted and fit as a function of the relaxation delay time using SPARKY routines. Steady-state NOE values are reported as the ratio of peak heights in paired spectra collected with and without an initial $4 \mathrm{~s}$ period of proton saturation during the $5 \mathrm{~s}$ recycling delay. For these studies, fragment and peptide concentrations were $325 \mu M$ and $1.1 \mathrm{mM}$, respectively.

\section{Fluorescence and circular dichroism measurements}

Steady-state fluorescence measurements were performed in a Jasco FP-6500 spectrofluorometer operating in the ratio mode and equipped with a thermostated cell 
holder connected to a circulating water bath set at $20^{\circ} \mathrm{C}$. A $0.3-\mathrm{cm}$ path length cell sealed with a Teflon cap was used. When the intrinsic fluorescence of proteins was measured, excitation wavelength was $295 \mathrm{~nm}$ and emission data were collected in the range 310-450 $\mathrm{nm}$. The spectral slit-widths were set to $3 \mathrm{~nm}$ for both monochromators. The protein concentration was $30 \mu \mathrm{M}$.

Circular dichroism measurements were carried out with a Jasco J-810 spectropolarimeter calibrated with (+) 10camphor sulfonic acid. Near-UV circular dichroism (CD) spectra were collected in $20 \mathrm{mM}$ TrisHCl buffer, $100 \mathrm{mM}$ $\mathrm{NaCl}, 1.0 \mathrm{mM}$ DTT, and $0.1 \mathrm{mM}$ ethylenediaminetetraacetic acid at $\mathrm{pH}$ 7.0. A cell of $1.0 \mathrm{~cm}$ path-length was used. Data were acquired at a scan speed of $20 \mathrm{~nm} \mathrm{~min}{ }^{-1}$, and at least three scans were averaged. Finally, blank (buffer) scans were subtracted from the spectra, and values of ellipticity were directly expressed as $\mathrm{mDeg}$. The protein concentration was $30 \mu \mathrm{M}$.

\section{Molecular dynamics simulations}

To investigate the molecular dynamics of fragment TRX1-93 and complex TRX94-108/TRX1-93, we carried out simulations with the GROMOS $53 \mathrm{a} 6$ force field 62 implemented in GROMACS 3.3.63 In all cases, the initial structures were generated from the coordinates of chain A in the crystallographic structure 2TRX. The structure of wild-type TRX, the fragment TRX1-93 or the complex TRX94-108/TRX1-93 was embedded in a dodecahedral periodic cell (90 or $114 \mathrm{~nm}^{3}$, respectively) and solvated with simple point charge water molecules. ${ }^{64}$ Sodium and chloride ions were added to neutralize charges in the peptides and to simulate a $150 \mathrm{mM}$ salt concentration. Conditioning consisted of 1000 steps of energy minimization and subsequent linear heating (from 0 to $300 \mathrm{~K}$ in $10 \mathrm{ps}$ ) by positionrestrained MDS. The system was simulated as an isothermal $(300 \mathrm{~K})$ /isobaric ( 1 bar) ensemble, using weak temperature and pressure coupling ( 0.1 and $1.0 \mathrm{ps}^{-1}$, respectively) ${ }^{65}$ All hydrogen atoms were explicitly considered. The masses of the hydroxyl hydrogen atoms were increased fourfold at the expense of the oxygen atom masses. The positions of the remaining hydrogen atoms were calculated as virtual interaction sites. ${ }^{66}$ All protein covalent bonds were constrained by LINCS. ${ }^{67}$ This setup allowed us to use a 4 -fs time-step, according to the work of Feenstra et al. ${ }^{68}$ Cut-off distances for neighbor searching, Lennard-Jones and Coulombic interactions were 9,9 , and $14 \AA$, respectively. Long-range interactions were computed according to the generalized reaction field method proposed by Tironi et al. ${ }^{69}$ Cluster analysis was carried out using the method of Daura et al. 70 Root mean square fluctuations (RMSF) were calculated for $\mathrm{C} \alpha$ atoms. For any geometrical measurements, the initial 20 ns of all simulations were discarded, so that assessments were made over those snapshots corresponding to the rootmean-square deviation (RMSD) plateau. The RMSF values were calculated using Eq. (3):

$$
\mathrm{RMSF}_{i}=\left(\frac{\sum_{s=1}^{N}\left(x_{s i}-\bar{x}_{i}\right)^{2}}{N}\right)^{1 / 2}
$$

where $x_{s i}$ stands for the $\mathrm{C} \alpha$ atoms coordinates of the $i$ th residue at snapshot $\mathrm{s}$, and $\overline{x_{i}}$ represents the average coordinates of the $i$ th residue along a trajectory of $N$ snapshots. The hydrodynamic radii of the protein variants were calculated assuming globular shape and by using $R_{\mathrm{g}} / R_{\mathrm{h}}=0.77$ along the simulations. ${ }^{57}$ In addition, $R_{\mathrm{h}}$ values were measured on particular snapshots taken along the simulations using HYDROPRO software. ${ }^{71}$

\section{RESULTS}

Mutual recognition between peptide TRX94108 and fragment TRX $1-93$ brings about $a$ native-like signature for the complex

The ${ }^{1} \mathrm{H}-{ }^{15} \mathrm{~N}$ heteronuclear single-quantum coherence (HSQC) spectrum of uniformly ${ }^{15} \mathrm{~N}$-labeled fragment TRX1-93 recorded at $20^{\circ} \mathrm{C}$ and $\mathrm{pH} 7.3$ is shown in Figure 1. Only a few ${ }^{1} \mathrm{H}-{ }^{15} \mathrm{~N}$ cross-peaks are evident, showing limited dispersion of their chemical shift values, a fact suggestive of the presence of unfolded oligomeric species in the sample (see the DOSY-NMR experiments below). A remarkable change in the HSQC spectrum, characterized by a narrowing and increased dispersion of chemical shifts values of the NMR signals, is observed when partially folded fragment ${ }^{15} \mathrm{~N}$-TRX1-93 is mixed with unlabeled peptide TRX94-108, indicating the appearance of a higher extent of structure. Concomitantly, a progressive increment in intensities of the ${ }^{1} \mathrm{H}-{ }^{15} \mathrm{~N}$ cross-peaks is also observed (Figs. 1 and 2 and Supporting Information Fig. S1). After an incubation of $4 \mathrm{~h}$ at $20^{\circ} \mathrm{C}$ in a SOFAST HMQC Real-Time 2D NMR experiment, 56 the resulting spectrum (Fig. 3 and Supporting Information Fig. S2) is similar to that of fulllength TRX, ${ }^{49}$ except for localized changes mapping to the loop connecting strand $\beta 2$ to helix $\alpha 2$, helix $\alpha 2$ and up to the N-terminus of helix $\alpha 3$ (stretch I23-I42, Supporting Information Fig. S2), a region including the active site of the protein. Thus, the lower enzymatic activity exhibited by the complex $(\sim 7 \%)$ in comparison to full-length TRX may result from a local perturbation of the active site. ${ }^{47,49}$ Subtle differences in the precise position of side chains of amino acid residues belonging to or in the vicinity of the active site (see below) could bear a dramatic influence on enzymatic activity.

We completed the backbone resonance assignments for ${ }^{13} \mathrm{C}-{ }^{15} \mathrm{~N}$ uniformly labeled fragment TRX1-93 in complex with unlabeled peptide TRX94-108 under reducing conditions, using standard heteronuclear NMR experiments. The assigned ${ }^{1} \mathrm{H}-{ }^{15} \mathrm{~N}$ HSQC is shown in Figure 3 (available as Supporting Information Table S2). Most importantly, the preparation of this sample involved the refold- 


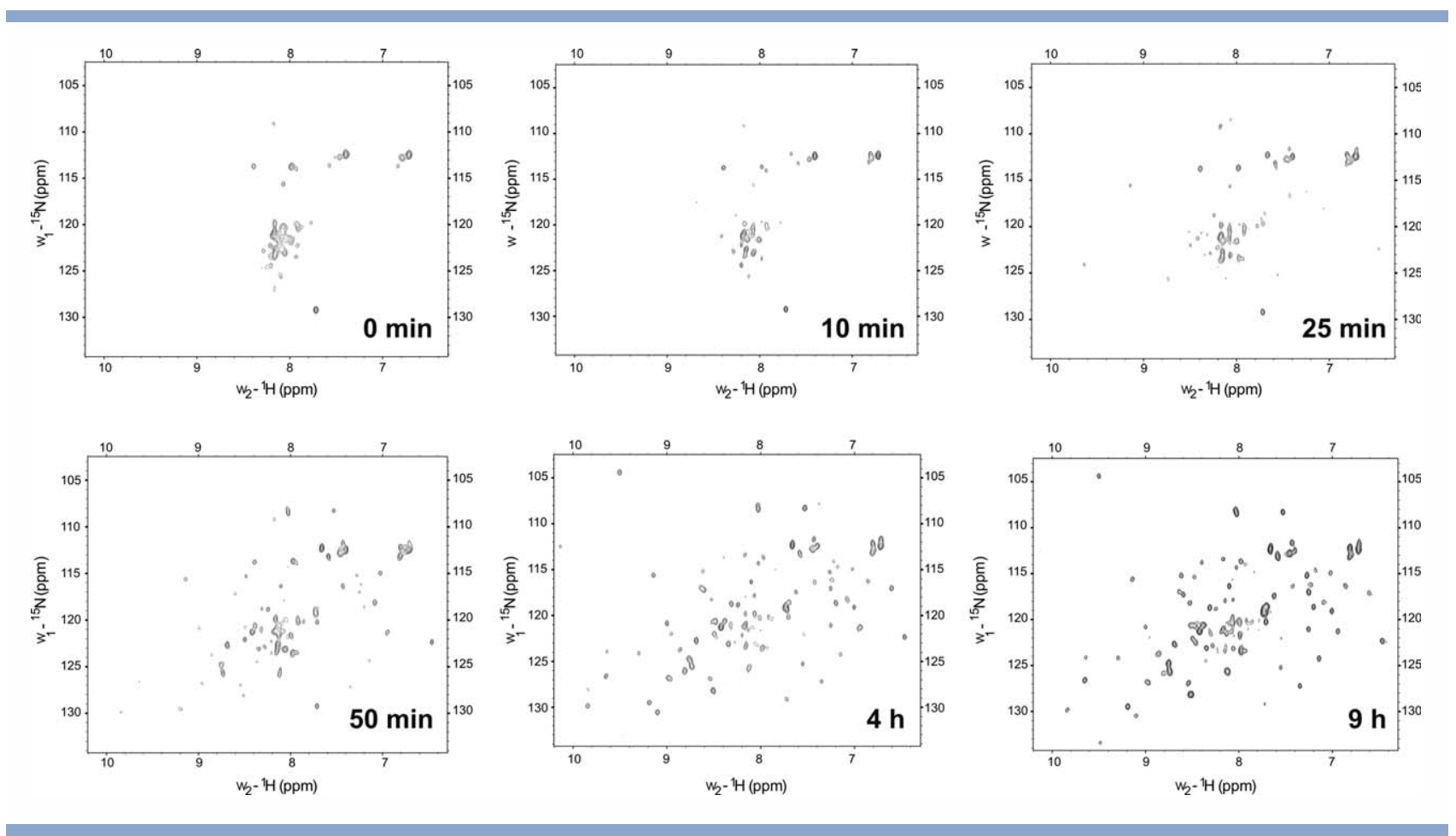

Figure 1

Time-course of complex consolidation on the interaction between fragment TRX1-93 and peptide TRX94-108. For visualization purposes, only six spectra of 85 are shown. Data collection for each spectrum takes $7 \mathrm{~min} 7 \mathrm{~s}$. The full experiments was run at $20^{\circ} \mathrm{C}$ in $20 \mathrm{mM}$ TrisHCl buffer, 100 $\mathrm{m} M \mathrm{NaCl}, 1.0 \mathrm{mM}$ DTT, and $\mathrm{pH}$ 7.3. Fragment and peptide concentrations are 100 and $333 \mu M$, respectively.

ing of fragment TRX1-93 at low concentration $(50 \mu M)$ at $20^{\circ} \mathrm{C}$ in the presence of peptide TRX94-108 $(250 \mu \mathrm{M})$. Afterwards, the complex was concentrated to $250 \mu \mathrm{L}$ by ultra filtration $(500 \mu \mathrm{M}$ final concentration of fragment TRX1-93). Under these conditions, protein aggregation along the refolding process is minimized, as attested by the absence of significant turbidity at the end of this procedure (as indicated by the low light scattering of the sample in the range $320-400 \mathrm{~nm}$ ). Most every ${ }^{1} \mathrm{H}-{ }^{15} \mathrm{~N}$ resonance is observed in the HSQC spectrum, except for those corresponding to amino acid residues D2 and G33. The former observation was also reported by others for the full-length protein. ${ }^{40,41}$ Furthermore, discrete heterogeneity at the C-terminal end involving residues G92 and A93, related to the presence of at least two different local conformations, becomes evident by the existence of two ${ }^{1} \mathrm{H}-{ }^{15} \mathrm{~N}$ cross-peaks for each residue (see G92* and A93* spots in Fig. 3). This behavior is more likely due to two possible different orientations of residues G92 and A93 when peptide TRX94-108 is attached to the fragment. This conformational heterogeneity is limited to the C-terminal vicinity, as attested by the reduction in the intensity of the second population $\left({ }^{*}\right)$ for residue G92 and the absence of a second cross-peak for residue V91.

In addition to the NMR evidence, optical spectroscopies support the acquisition of global native-like secondary and tertiary structure as a consequence of peptide/frag- ment interaction. Thus, isolated fragment TRX1-93 is characterized by a non-native emission fluorescence spectrum where intensity is less quenched than in the complexed form [Fig. 4(A)]. Nevertheless, tryptophan residues still exhibit a relatively apolar environment in the isolated fragment (fluorescence emission $\lambda_{\operatorname{MAX}}=340$ $\mathrm{nm})$. In addition, the near-UV CD signals of the fragment is almost absent, most likely pointing to a mobile environment around aromatic residues [Fig. 4(B)]. Moreover, the far-UV CD spectrum of the isolated fragment is compatible with the presence of residual secondary structure, a signature of highly flexible states [see Fig. 2(A) in Ref. ${ }^{47}$ ]. Importantly, on peptide addition, the interaction brings about the appearance of a global native-like secondary structure signature and the near-UV CD spectrum also becomes indistinguishable from that of native TRX. These observations prompted us to advance the study of the kinetics of this peptide/protein association. To attain this goal, manual mixing was adequate, given the slow rate of this process. The evolution of the far and near-UV signals (measured in the range $220-230 \mathrm{~nm}$ and 275-285, respectively) is shown in Figure 4(C,D). In both cases, the kinetics is well described by two exponential functions: $k_{1}=0.34 \pm 0.02 \mathrm{~min}^{-1}$ and $k_{2}=0.0317$ $\pm 0.0007 \mathrm{~min}^{-1}$ in the far-UV region, and $k_{1}=0.25 \pm$ $0.01 \mathrm{~min}^{-1}$ and $k_{2}=0.0213 \pm 0.0005 \mathrm{~min}^{-1}$ in near-UV region. It should be noted that the slowest process falls 

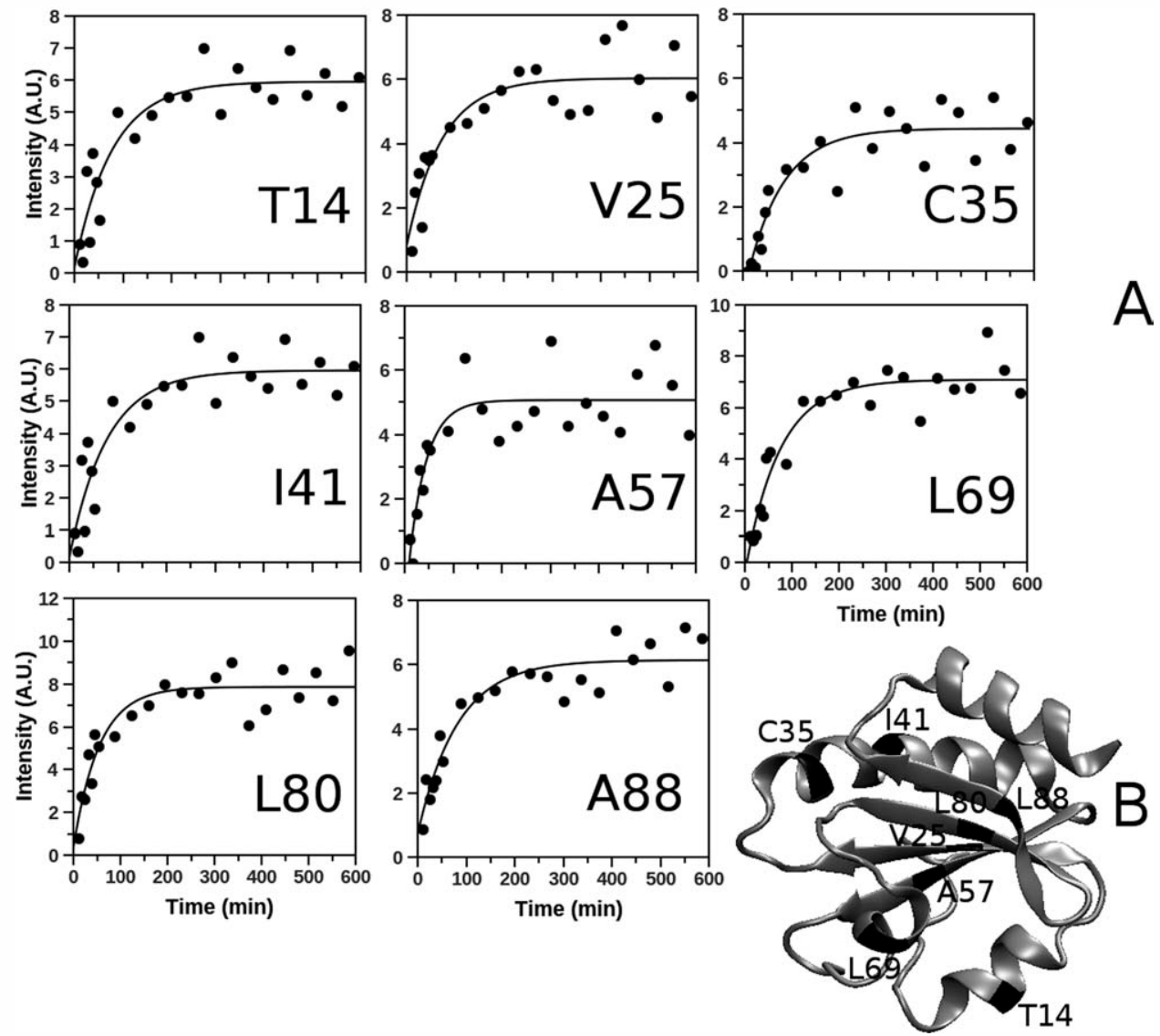

\section{Figure 2}

Reaction of complex TRX1-93/TRX94-108 formation followed by real-time NMR. (A) Intensities of ${ }^{1} \mathrm{H}-{ }^{15} \mathrm{~N}$ cross-peaks for residues, measured from ${ }^{1} \mathrm{H}-{ }^{15} \mathrm{~N}$ HET-SOFAST experiments run under the experimental conditions indicated in Figure 1 were plotted as a function of reaction time $(t$ $=0$ being the time when mixing of peptide and fragment occurs). For visualization purposes, only the behavior of eight selected residues (T14, V25, C35, I41, A57, L69, L80, and A88) is shown here (for the full graphics, see Supporting Information Fig. S1 and Table S3). Each curve could be accounted for by one exponential component. Within experimental error, all intensity curves follow similar kinetics, yielding an average value of $k$ $=0.016 \pm 0.009 \mathrm{~min}^{-1}\left(t_{1 / 2}=42 \mathrm{~min}\right)$. (B) Ribbon representation of TRX (PDB ID: 2TRX) where residues mentioned before are shown in black.

in the same time scale as that observed by the change in ${ }^{1} \mathrm{H}-{ }^{15} \mathrm{~N}$ amide intensities, characterized by an average value of $k=0.016 \pm 0.009 \mathrm{~min}^{-1}$.

We also probed the stability of the end product by fluorescence spectroscopy [inset to Fig. 4(A)]. Significantly, the TRX complex experiences a urea-unfolding transition $\left(\Delta G_{\mathrm{NU} \mathrm{H} 20}=1.86 \mathrm{kcal} \mathrm{mol}^{-1}, m_{\mathrm{NU}}=1.28 \mathrm{kcal} \mathrm{mol}^{-1}\right.$ $\left.M^{-1}, C_{\mathrm{m}}=1.44 M\right)$ characterized by intrinsic fluorescence by an increment of intensity and a red shift of the wavelength maximum $\left(\lambda_{\mathrm{MAX}}\right)$. Thus, the complex is significant destabilized $\left(\sim 4 \mathrm{kcal} \mathrm{mol}^{-1}\right)$ in comparison to the reduced form of full-length TRX. 47

\section{The complex adopts a compact structure exhibiting native-like secondary structure}

To evaluate the compactness of TRX species, we studied by DOSY-NMR the hydrodynamic behavior of fragment TRX1-93 when it is refolded in the presence or in the absence of peptide TRX94-108 (Table I). In the absence of the latter, fragment TRX1-93 shows a remarkable tendency to aggregate at high protein concentration. Isolated fragment TRX1-93 at $100 \mu \mathrm{M}$ yields an $R_{\mathrm{h}}$ value of $33.9 \AA$, compatible with an aggregated state. By assuming a globular shape, this value matches with six to seven molecules of fragment per aggregate particle. However, when refolding is performed in the presence of the peptide, the size of the complex $(18.2 \AA)$ turns to be compatible with that of full-length TRX, indicating the existence of a transition from a partially folded and aggregated form toward a compact monomer.

In addition, to characterize the overall compactness of the complex, we performed 2D ${ }^{1} \mathrm{H}-{ }^{15} \mathrm{~N}$ HET-SOFASTHMQC experiments. Here, the ${ }^{1} \mathrm{H}-{ }^{1} \mathrm{H}$ spin diffusion (NOE) from aliphatic to amide groups is quantified by $\lambda_{\mathrm{NOE}}$ (Fig. 5). ${ }^{58}$ In general, values of this parameter fluctuate between 0.3 for well-folded globular proteins 


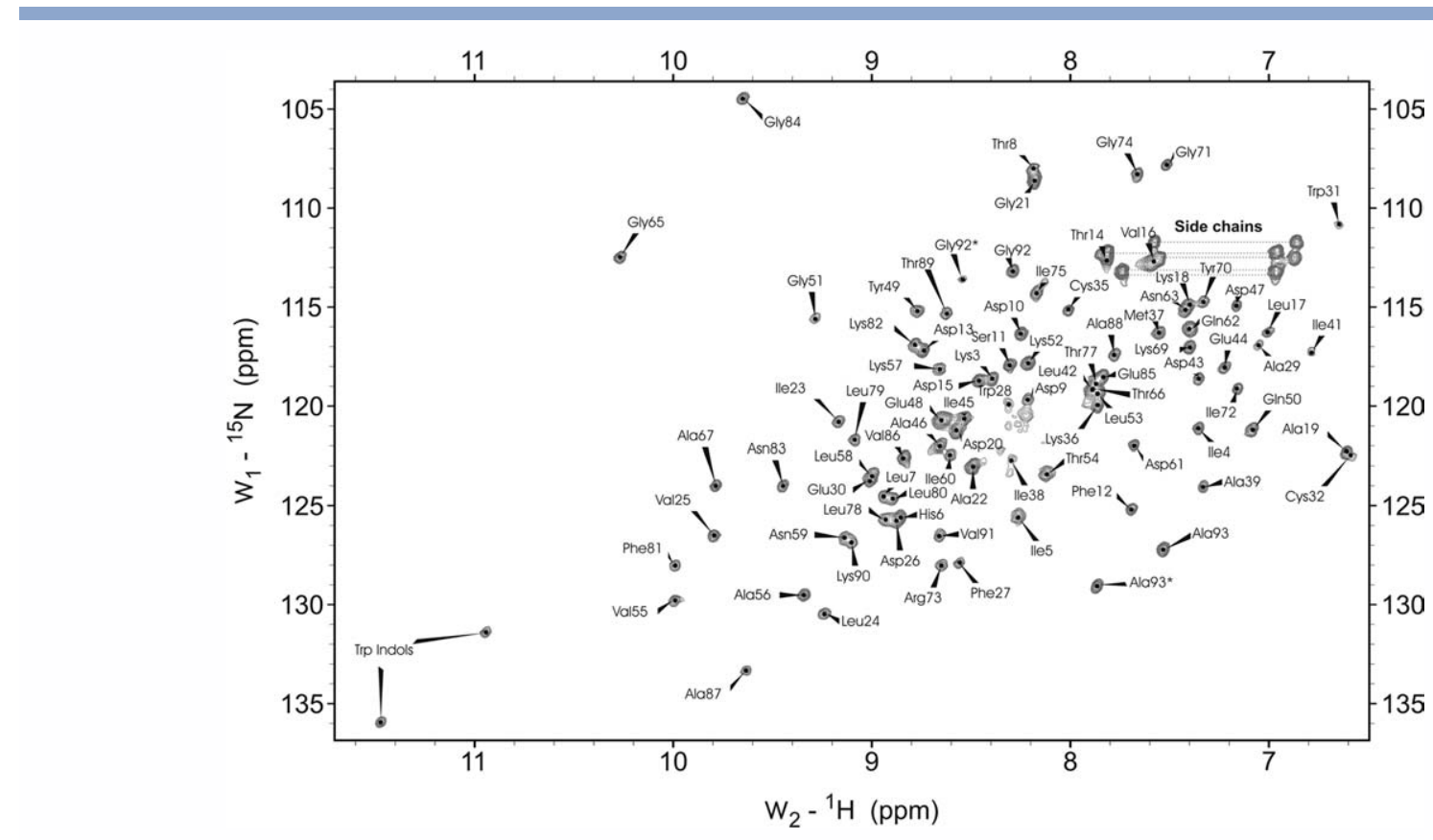

Figure 3

Backbone assignment of reduced E. coli fragment TRX1-93 in complex with peptide TRX94-108. The concentration of ${ }^{15} \mathrm{~N}-l$ labeled or ${ }^{13} \mathrm{C}-{ }^{15} \mathrm{~N}-$ labeled fragment TRX1-93 is $500 \mu \mathrm{M}$. Unlabeled peptide TRX94-108 is present at $1.5 \mathrm{mM}$ concentration. All experiments were run at $20^{\circ} \mathrm{C}$ in 20 $\mathrm{m} M$ TrisHCl buffer, $100 \mathrm{mM} \mathrm{NaCl}, 1.0 \mathrm{mM}$ DTT, and $\mathrm{pH}$ 7.3.

and 0.6 for mostly unfolded (random coil) polypeptides. 58 TRX1-93 in complex with the peptide shows a mean $\lambda_{\mathrm{NOE}}$ of 0.2 , a value similar to that observed for full-length TRX. Furthermore, it is interesting to note that there are no residues with $\lambda_{\mathrm{NOE}}>0.5$ and that the region corresponding to residues D20, G21, and A22, with $\lambda_{\mathrm{NOE}}$ values of $\sim 0.3$ and $\sim 0.4$ for the complex and full-length protein, respectively, maps to the exposed loop connecting helix $\alpha 1$ and strand $\beta 1$. These results denote that after interaction with peptide TRX94-108, fragment TRX1-93 is able to acquire a well-folded state. In this work, we also took advantage of the high sensitivity of NMR chemical shift information to infer secondary structure. ${ }^{72,73}$ In particular, from resonances corresponding to $H \alpha, C \alpha$, and $C^{\prime}$, it is possible to draw insights on the structure of the complex. Briefly, the deviation of the observed chemical shifts from consensus random coil values (secondary shifts $\Delta \delta$ ) is indicative of secondary structure order. Negative or positive deviations for $\mathrm{H} \alpha$ atoms, suggest that the backbone adopts $\alpha$-helical or $\beta$-strand conformation, respectively. In addition, negative or positive deviations in $\mathrm{C} \alpha$ and $\mathrm{C}^{\prime}$ shifts are indicative of $\beta$ or $\alpha$ organization, respectively. A residue-specific analysis for the complex revealed the occurrence of a general pattern of secondary structural elements similar to that observed for the wild-type protein (Fig. 6).

Interestingly, $\mathrm{C} \alpha$ and $\mathrm{C} \beta$ secondary shifts for fulllength TRX and for the complex are fully super imposable indicating that the structural features of the complex strongly resemble those of the full-length protein (Supporting Information Fig. S3). Furthermore, we measured three-bond $\mathrm{HNH} \alpha$ coupling constants $\left({ }^{3} \mathrm{~J}_{\mathrm{HNH} \alpha}\right)$, a helpful indicator of the torsion angle $\Phi$ populated by each residue. ${ }^{54-56}$ This coupling falls in the range 3-5 $\mathrm{Hz}$ for an $\alpha$ helix and $8-11 \mathrm{~Hz}$ for a $\beta$-sheet structure. For a random-coil, a weighted average of these values is observed, that typically ranges between 6.0 and $8.0 \mathrm{~Hz}$ for most residues. The deviations of the observed coupling constants from the random-coil values $\left(J_{\mathrm{obs}}-J_{\mathrm{rc}}\right.$, the secondary coupling constants) give valuable information on the secondary structural propensities along the backbone as well. Negative or positive values indicate helical or $\beta$-sheet propensities, respectively. Secondary ${ }^{3} J_{\mathrm{HNH} \alpha}$ coupling values are shown in Figure 6(D). As a whole, our results suggest that, after peptide binding, the fragment acquires a conformation compatible with a secondary structure pattern keeping a close resemblance to that observed for native TRX, in full agreement with the ${ }^{13} \mathrm{C}$ and ${ }^{1} \mathrm{H}$ secondary chemical shifts measured.

\section{Titration of fragment TRX1-93 with peptide TRX94-108 also reveals site-specific structural changes}

We performed an equilibrium-titration experiment of fragment TRX1-93 with peptide TRX94-108 to determine the dissociation constant of the process and to sample 

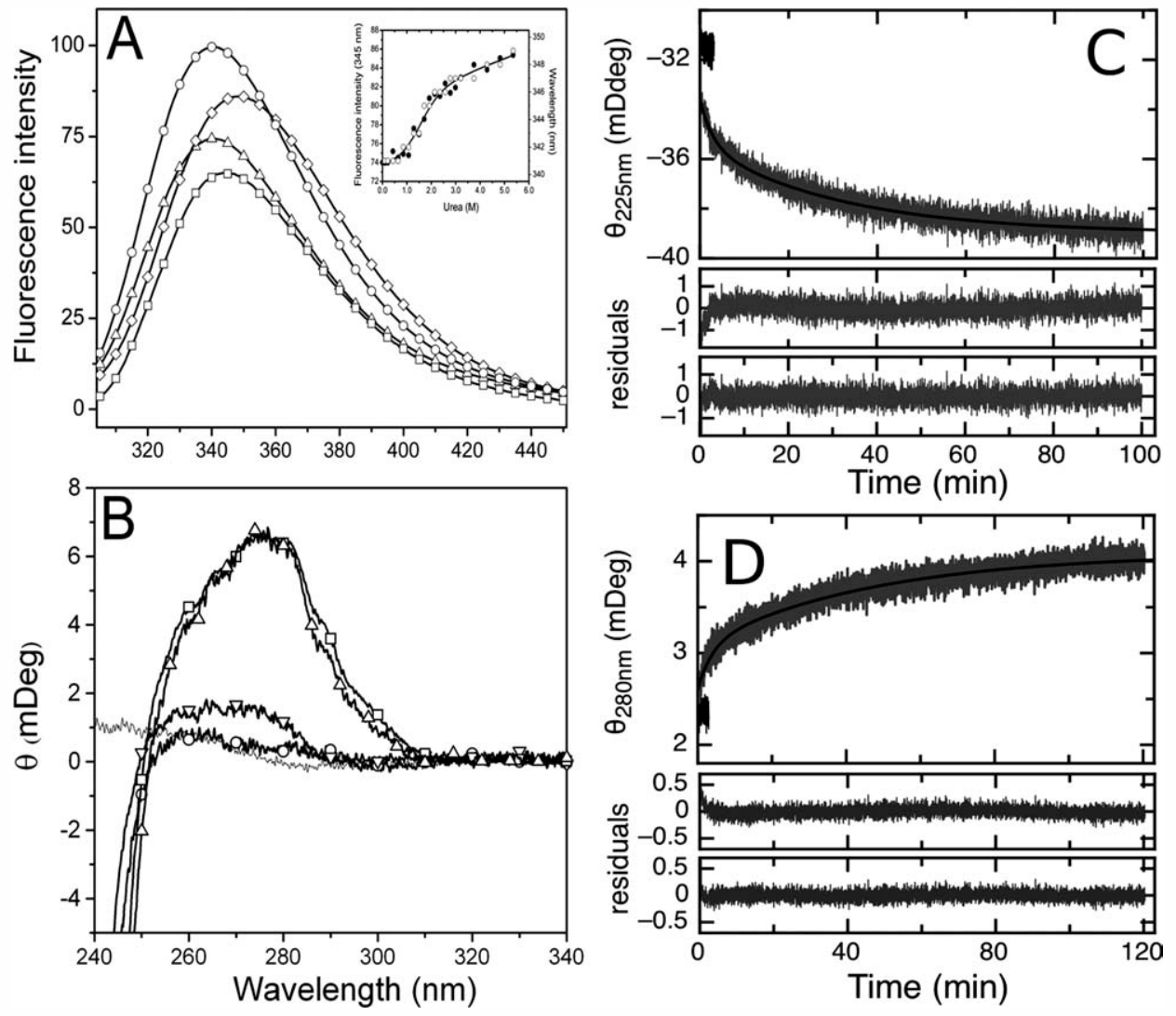

\section{Figure 4}

The tryptophan fluorescence emission $(\mathbf{A})$ and near-UV CD $(\mathbf{B})$ spectra are shown for isolated fragment TRX1-93 $(\bigcirc)$, the complex $(\triangle)$, and the reduced form of full-length E. coli TRX in the native state $(\square)$. In panel A, the unfolded complex in $5 M$ urea $(\diamond)$ is also shown as a reference, and the inset corresponds to the urea-induced unfolding of complex TRX1-93/TRX94-108. The transition was followed by the change in fluorescence intensity of tryptophan emission at $345 \mathrm{~nm}(-)$ and the shift of the maximal emission wavelength $\left(\lambda_{\mathrm{MAX}}, \bigcirc\right)$, plotted as a function of urea concentration. In panel B, the near-UV CD spectra of the isolated peptide TRX94-108 ( $\nabla)$ and buffer baseline $(-)$ are also shown. Fragment concentration was $30 \mu \mathrm{M}$ and a 3:1 peptide to fragment molar ratio was used. Time-course of complex formation followed by CD spectroscopy in the far- (C) and near-UV (D) regions. After regression analysis, the fitted curves are plotted that consider two exponential components, together with the residual errors for one and two exponential components (upper and lower panels, respectively). The values of the kinetic constants are reported in the main text. The observed changes in amplitude account for 90 and $72 \%$ of the equilibrium values, for the near- and far-UV regions, respectively. A bandwidth of $10 \mathrm{~nm}$ was set, centered at 225 and $280 \mathrm{~nm}$ for the far- and near-UV regions, respectively. The data pitch was $1 \mathrm{~s}$ for a total acquisition time of $120 \mathrm{~min}$. Fragment and peptide final concentrations were 84 and $280 \mu \mathrm{M}$, respectively. All experiments were run at $20^{\circ} \mathrm{C}$ in $20 \mathrm{~m} M$ TrisHCl buffer, $100 \mathrm{mM} \mathrm{NaCl}, 1.0 \mathrm{mM}$ DTT, and $\mathrm{pH} 7.3$.

the changing environments due to the structural consolidation of the complex. As judged by the analysis of cross-peak heights as a function of peptide/fragment ratio (inset in Fig. 7) the dissociation equilibrium is characterized by a $K_{\mathrm{D}}$ value of $1.4 \pm 1.0 \mu M$. This result is in good agreement with the values previously measured by isothermal titration calorimetry (ITC) and near-UV CD (see Supporting Information Table S1). Noticeably, in a peptide to fragment molar excess ratio 1.0-3.5, most ${ }^{1} \mathrm{H}-{ }^{15} \mathrm{~N}$ resonances do not experience significant changes in chemical shift. However, a subgroup of ${ }^{1} \mathrm{H}-{ }^{15} \mathrm{~N}$ signals (nine resonances) shows a gradual change in chemical shift, likely revealing the existence of a fast exchange regime (Fig. 7 and Supporting Information Fig. S4). The residues involved include amino acids from the $\mathrm{N}$ - and
C- termini (I4, V91, G92, and A93), the core residue F27, residues located near the active site (W31, C35, and A39), and residue I41, directly interacting with $\alpha$-helix 5 that is represented in the complex by peptide TRX94-108 [Fig. $7(\mathrm{C})]$. The fact that most resonances remain unchanged in regard to their combined chemical shift positions supports the view that the complex globally behaves as a cooperative folding unit. Nevertheless, the gradual change in chemical shift observed for the above mentioned subset of residues might result from a second lower affinity binding event. This would be characterized by a $K_{\mathrm{D}}$ value higher than $\sim 150 \mu M$, according to the progress of the chemical shifts measured as a function of peptide to protein molar excess (Supporting Information Figs. S4 and S5). Although subtle conformational rear- 
Table I

Hydrodynamic Radii $\left(\mathrm{R}_{\mathrm{h}}\right)$ of Different TRX Species Determined by Diffusion Order Spectroscopy (DOSY) and Molecular Dynamics Simulations (MDS)

\begin{tabular}{lcc} 
TRX Species $^{\mathrm{a}}$ & $R_{\mathrm{h}}(\AA)^{\mathrm{b}}$ & Peptide/fragment \\
\hline DOSY & & \\
Full-length TRX $^{\mathrm{c}}$ & 17.9 & - \\
Peptide TRX94-108 $^{\mathrm{d}}$ & 10.2 & - \\
Fragment TRX1-93 $^{\mathrm{e}}$ & 33.9 & - \\
Complex TRX1-93/TRX94-108 $_{\text {Complex TRX1-93/TRX94-108 }}$ & 20.5 & 0.5 \\
Complex TRX1-93/TRX94-108 & 18.9 & 1.0 \\
MDS & 18.2 & 2.0 \\
Fragment TRX1-93 (monomer) & $17.1 \pm 0.3^{\mathrm{f}}$ & - \\
Complex TRX1-93/TRX94-108 & $16.6 \pm 0.1^{\mathrm{f}}$ & 1.0 \\
Full-length TRX & $16.6 \pm 0.1^{\dagger}$ & - \\
\end{tabular}

${ }^{a}$ Fragment TRX1-93 and full-length TRX concentrations were $100 \mu M$.

${ }^{\mathrm{b}}$ Uncertainties in the $R_{\mathrm{h}}$ values obtained by DOSY NMR were estimated by comparing the decay rates of the signals in the aromatic and aliphatic regions. In all cases differences of less than $5 \%$ were retrieved. This is in full agreement with the $R_{\mathrm{h}}$ values obtained by SEC-HPLC and DLS for the full-length TRX species.

cAssuming a globular shape, the theoretical $R_{\mathrm{h}}$ of TRX is $17.7 \AA$. The $R_{\mathrm{h}}$ calculated by SEC-HPLC is $18.0 \AA$ and a value of $16.8 \pm 3.0 \AA$ is estimated by dynamic light scattering (data not shown).

${ }^{\mathrm{d}}$ The isolated peptide is monomeric as judged by light scattering. ${ }^{30}$

${ }^{\text {e}}$ The dependence of the average hydrodynamic radius of fragment TRX1-93 with protein concentration was studied by SEC-FPLC. ${ }^{30}$ Values of 26 and $22 \AA$ at fragment concentrations of 81 and $16 \mu M$, respectively, were those therein reported.

${ }^{\mathrm{f}}$ The average hydrodynamic radius and the standard deviation of this value for each protein variant was calculated along the simulations, assuming globular shape and by using $R_{\mathrm{g}} / R_{\mathrm{h}}=0.77 .57$

rangements concomitant to this secondary binding event are not excluded, it is noteworthy to point out that the sites involved occur in the general neighborhood of the catalytic site of the protein (seven of nine residues lie at a distance shorter than $8.5 \AA$ of the CGPC motif).

\section{Backbone dynamics support the notion that the TRX complex adopts a well-folded tertiary structure}

Knowledge of $R_{1}, R_{2}$, and NOEs provides dynamical information about the internal motions in molecules over a wide range of time scales. Here, the question arises on whether-in the context of the complex-fragment TRX1-93 would be able to explore a significantly different structural space than that of full-length TRX. Indeed, we observed that the backbone resonances of the complex exhibit a relaxation behavior compatible with that expected for a native-like structure (Fig. 8). For comparison, we performed similar experiments with full-length TRX under the same experimental conditions $(\mathrm{pH} 7.3$, DTT $1.0 \mathrm{mM}$ ). Most residues of the complex and fulllength TRX show $R_{1}$ values in the ranges $1.81 \pm 0.13$ and $1.81 \pm 0.19 \mathrm{~s}^{-1}$, respectively. In addition, $R_{2}$ values fall in the range $10.3 \pm 1.8$ (complex) and $10.4 \pm 2.5$ $\mathrm{s}^{-1}$ (full-length TRX). Finally, most residue NOEs lie in the range $0.70 \pm 0.13$ (complex) and $0.76 \pm 0.15$ (fulllength TRX). Collectively, these values for backbone dynamics are fully compatible with those measured before at $\mathrm{pH} 5.7$ for full-length TRX. ${ }^{42}$ Overall, the relaxation data presented in this section, along with the secondary shifts and J-couplings analyzed previously, establishes not only that the interaction between fragment TRX1-93 and peptide TRX94-108 leads to a conserved structure similar to that of full-length TRX, but also that the complex would share native backbone dynamics. These data emphasize the prominent role played by segment 94-108 in triggering the consolidation of the TRX structure. However, a more detailed comparison of NOEs at the level of individual residues shows that the values for the complex are slightly lower than those for full-length TRX, indicating an enhanced dynamics (within the ps-ns range, those fast motions occurring close to the ps limit) for the complex. In addition, $R_{2}$ values for residues $43-$ 50 (helix $\alpha 3$ ) and $74-89$ ( $\beta$ hairpin at the C-terminus of the fragment are higher for the complex than for fulllength TRX). This fact would indicate a reduction in flexibility (a decrease of the slower motions, i.e., those in the ns regime) for this part of the complex and/or, more likely, the occurrence of an exchange process involving

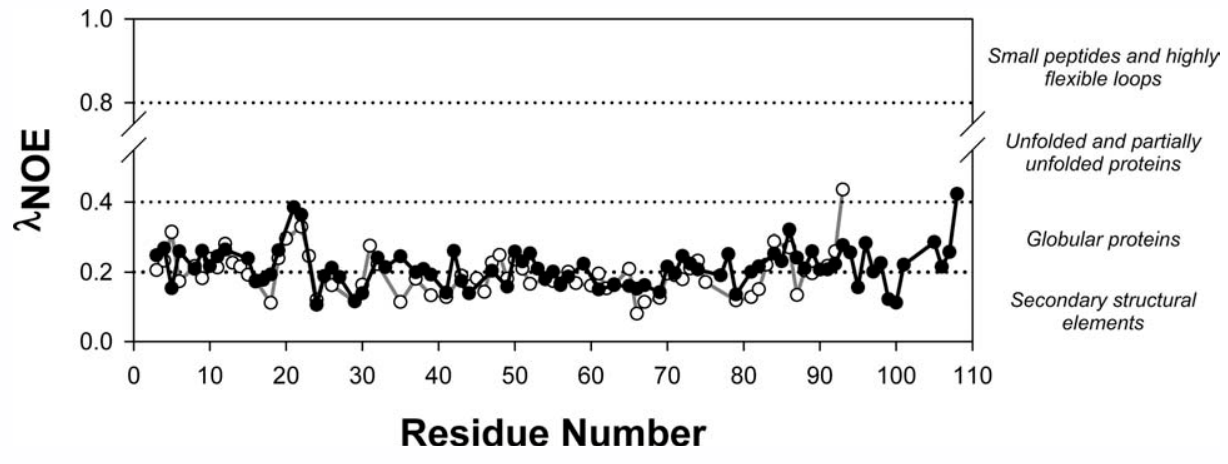

Figure 5

2D ${ }^{1} \mathrm{H}_{-1}{ }^{15} \mathrm{~N}$ HET-SOFAST-HMQC experiments on the complex TRX1-93/TRX94-108. $\lambda_{\text {NOE }}$ values for the complex are plotted along the sequence (O). The concentrations of fragment TRX1-93 and peptide TRX94-108 are 300 and $900 \mu M$, respectively. Full-length TRX in the native state (300 $\mu M)$ is shown as a control sample $(\bigcirc)$. For reference, the empirical threshold values of $\lambda_{\mathrm{NOE}} 58$ are shown on the right. For additional details, see Experimental Procedures. 


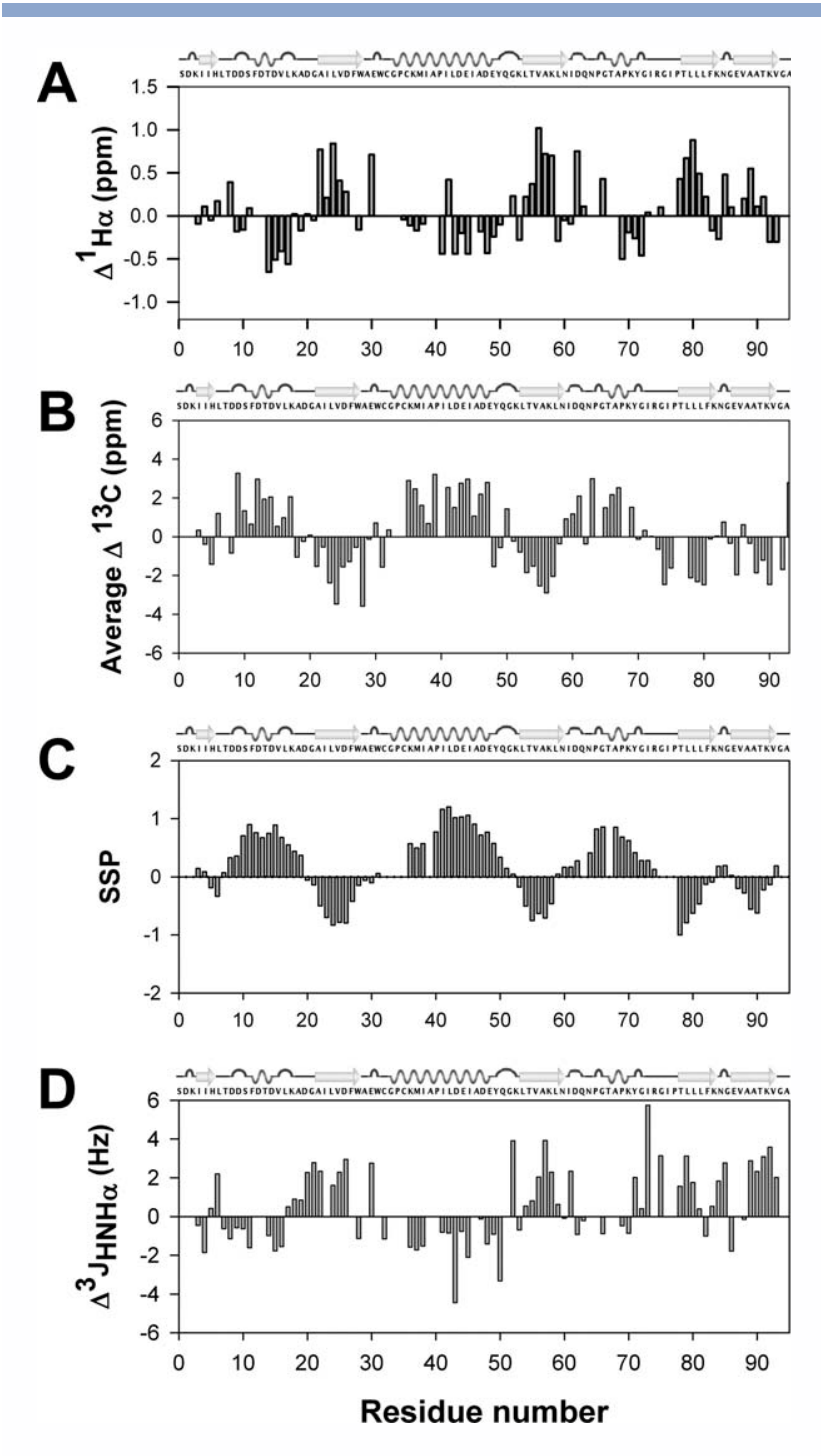

Figure 6

Secondary structure analysis of the complex TRX1-93/TRX94-108. (A) secondary shift values for $\mathrm{HA}$, (B) Average secondary shifts for ${ }^{13} \mathrm{C} \alpha$ and ${ }^{13} \mathrm{CO},(\mathbf{C})$ the secondary structure propensity score (SSP), ${ }^{53}$ (D) three bond $\mathrm{HNH} \alpha$ coupling constants $\left({ }^{3} \mathrm{~J}_{\mathrm{HNH} \alpha}\right)$. A secondary structure scheme for residues 1-93 taken from PDB ID: 2TRX is shown on top of each panel.

that region. Suggestively, this set of residues maps to the putative interacting surface with the cognate peptide TRX94-108 (Supporting Information Fig. S6).

\section{Molecular dynamics simulations provide insights into the conformational ensembles of fragment TRX1-93 and the TRX complex}

The high propensity of fragment TXR1-93 to form soluble aggregates at the protein concentration required for NMR experiments precludes the acquisition of clearcut site-specific information (see Fig. 1, time $=0 \mathrm{~min}$ ). However, the presence of residual secondary structure in the fragment is inferred by far-UV CD spectroscopy, where mainly the monomeric species is populated with respect to aggregated forms at low enough concentrations (Supporting Information Fig. S7). In this scenario, MDS offer a valuable tool to shed light on the conformation and dynamics of the monomeric fragment.

We ran all-atom MDS to draw a comparison between fragment TRX1-93 and the complex TRX94-108/TRX1-93. RMSD measurements along the simulations are shown in Figure 9. As judged by these results, the conformation of both the isolated fragment and the complex stabilizes in 20 ns or less. However, despite the limited time of these trajectories restricting the sampling of the conformational space, the absence of peptide TRX94-108 gives rise to important conformational changes (Figs. 9 and 10). Interestingly, on average the remainder polypeptide chain increases its mobility, as inferred by the analysis of fluctuations per residue [indicated by the higher RMSF values shown in Fig. 9(E)]. This behavior is more evident in regions mapping to the original helical elements. Thus, the secondary structure of the stretch 35-50, comprising helices $\alpha 2$ and $\alpha 3$ in fulllength TRX, is vastly perturbed, losing all helical character [Fig. 10(A,C,E)]. Nevertheless, the fluctuating remainder $\beta$ strands maintain general organizational features of the corresponding $\beta$-sheet present in native TRX. These results agree well with the experimentally observed molten globulelike character of the fragment, as evidenced by ANS binding, higher susceptibility to proteolytic cleavage as compared to the complex or full length TRX, ${ }^{49}$ the obliteration of nearUV CD signals (Fig. 4) and the almost featureless far-UV $\mathrm{CD}$ spectrum indicative of the absence of stable secondary structure (Supporting Information Fig. S7). In line with the evidence above, TRX1-93 does not exhibit a temperatureinduced cooperative transition. ${ }^{49}$ Despite the extensive loss of structure, isolated fragment TRX1-93 remains compact all along the simulations, as inferred from the invariance of its radius of gyration $\left[R_{\mathrm{g}}\right.$, Fig. $9(\mathrm{C})$ and $R_{\mathrm{h}}$, Table I]. This behavior agrees well with results from size-exclusion chromatography (SEC)-fast protein liquid chromatography (FPLC) experiments performed at low protein concentration, where the fragment behaves as a slightly expanded monomer. However, we cannot exclude the existence of further structural rearrangements brought about by the oligomerization of the fragment at high protein concentrations (Table I), which cannot be addressed by classic all-atom MDS.

By contrast, fragment TRX1-93 in complex with peptide TRX94-108 exhibits a general native-like conformation, prominently including the stabilization of helices $\alpha 2$ and $\alpha 3$ [comprised in the stretch 35-50, Fig. $10(\mathrm{~B}, \mathrm{D}, \mathrm{F})]$ and a decrease of the average polypeptide chain mobility, as shown by the analysis of RMSF values [Fig. 9(F)]. This inference is supported by near-UV CD and NMR results, suggesting the acquisition of a nativelike tertiary structure of the fragment exhibiting restricted mobility when embedded in the context of the 

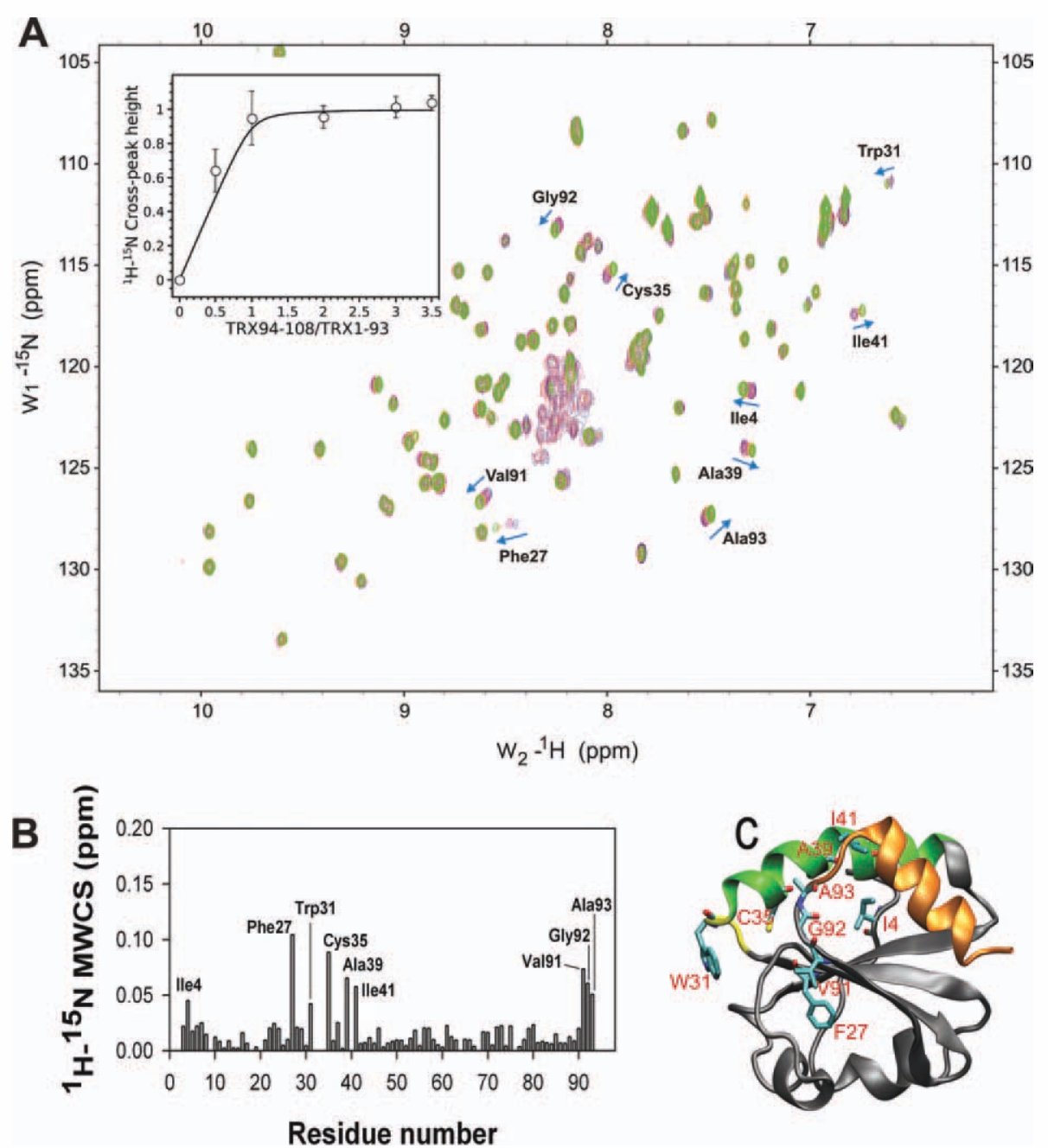

Figure 7

Titration of ${ }^{15} \mathrm{~N}$ uniformly labeled fragment TRX1-93 with unlabeled peptide TRX94-108. (A) Dialyzed fragment ${ }^{15} \mathrm{~N}-\mathrm{TRX1}-93$ (100 $\mu \mathrm{M}$, final concentration) was mixed with peptide TRX94-108 (0.5:1, blue; 1:1, brown; 2:1, purple 3:1, orange; and 3.5:1, green, molar ratio of peptide to fragment). ${ }^{1} \mathrm{H}-{ }^{15} \mathrm{~N}-\mathrm{HSQC}$ spectra were acquired after at least $16 \mathrm{~h}$ of incubation at room temperature. Inset: Analysis of cross-peak heights as a function of peptide/fragment molar ratio. For the calculation, 12 well-resolved cross-peaks were selected from each HSQC spectrum. After normalization of peak heights by the maximal value measured for each residue, the mean and standard deviation for each peptide/fragment ratio was plotted. An identical analysis carried out with peak volumes yields similar results (not shown). The black line represents the fitting to the data of model considering a 1:1 peptide/fragment stoichiometry. (B) Mean-weighted ${ }^{1} \mathrm{H}-{ }^{15} \mathrm{~N}$ chemical shifts (MWCS in ppm) as a function of residue number. Normalized MWCS upon peptide binding (3.5:1) were calculated using ${ }^{1} \mathrm{H}-{ }^{15} \mathrm{~N}$ MWCS $=\left[\Delta H^{2}+(\Delta N / 5)^{2}\right]^{1 / 2}$, where $\Delta H$ and $\Delta N$ are the differences in chemical shift for ${ }^{1} \mathrm{H}$ and ${ }^{15} \mathrm{~N}$ observed between the complex at 0.5:1 and at 3.5:1, molar relations peptide to fragment. (C) Ribbon diagram of full-length E. coli TRX. Residues that experience significant change in chemical shift are represented in licorice. The $\alpha 2 / \alpha 3$ structural element (green), the connector loop (yellow), between the former and the $\beta 2$ element and C-terminal helix comprising residues 94-108, (green) are highlight in colors. Molecular graphics were prepared using visual molecular dynamics. ${ }^{74}$

complex [Fig. 9(F)].Overall, MDS show that the complex appears as compact as the full-length protein (Table I), a fact that agrees well with all the available experimental evidence. For the sake of comparison, we also ran MDS for full-length TRX. Interestingly, these simulations show that C $\alpha$ RMSF values for wild-type TRX are slightly lower than those for the complex [Fig. 9(G)]. This behavior might reveal a slightly enhanced flexibility for the latter, in agreement with the lower NOE measure- ments observed. In particular, MDS of the complex show the largest differences in C $\alpha$ RMSF values with respect to full-length TRX for helical elements $\alpha 1$ and $\alpha 4$.

\section{DIscussion}

Interaction between fragment TRX1-93 and peptide TRX94-108 triggers the consolidation of a native-like complex. Along this process, fragment and peptide expe- 


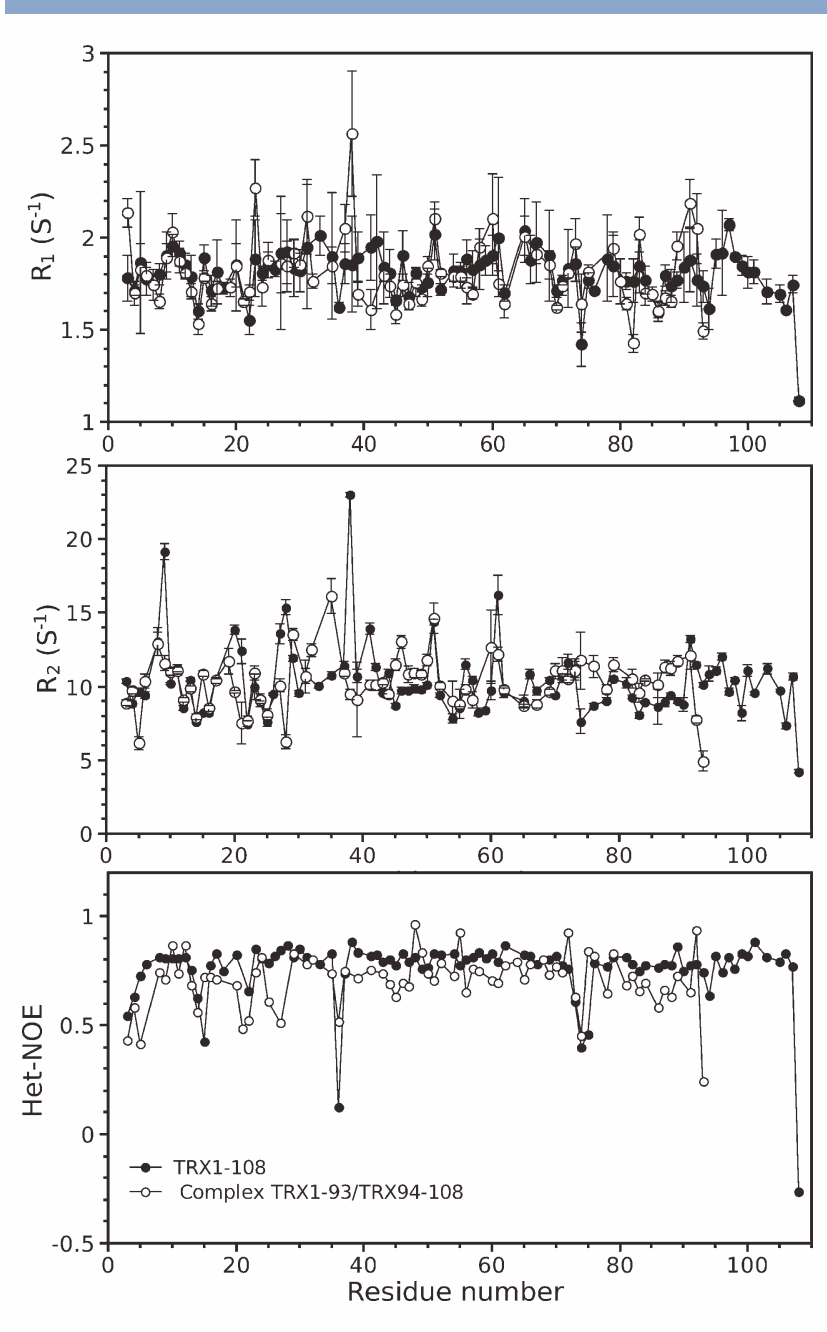

Figure 8

Backbone dynamics of the complex TRX1-93/TRX94-108. The dynamics of the reduced form of fragment TRX1-93 refolded in the presence of peptide TRX94-108 is shown $(\bigcirc)$. For comparison, the corresponding parameters for the reduced form of full-length E. coli TRX were measured under the same experimental conditions $(\bigcirc)$. Experiments to obtain $R_{1}, R_{2}$, and steady-state heteronuclear NOE values were performed as described in Materials and Methods. All experiments were run at $20^{\circ} \mathrm{C}$ in $20 \mathrm{mM}$ Tris $\mathrm{HCl}$ buffer, $100 \mathrm{mM} \mathrm{NaCl}, 1.0 \mathrm{mM}$ DTT, and $\mathrm{pH}$ 7.3. The concentrations of fragment TRX1-93 and peptide TRX94-108 are $325 \mu M$ and $1.1 \mathrm{mM}$, respectively. Full-length TRX is at $450 \mu \mathrm{M}$. For $R_{1}, R_{2}$, and heteronuclear NOE calculation only isolated cross-peaks were used.

rience a disorder-to-order transition. Here, molecular recognition, folding and structure stabilization take place. Even though the stability of the resulting structure is substantially diminished by comparison with the reduced form of the full-length protein, ${ }^{77}$ this complex is able to acquire fine structural features characteristic of TRX. From the NMR data herein presented, we infer that the conformational specificity encrypted in this interaction alters the energy gap between non-native and native conformations populated by isolated fragment TRX1-93, so
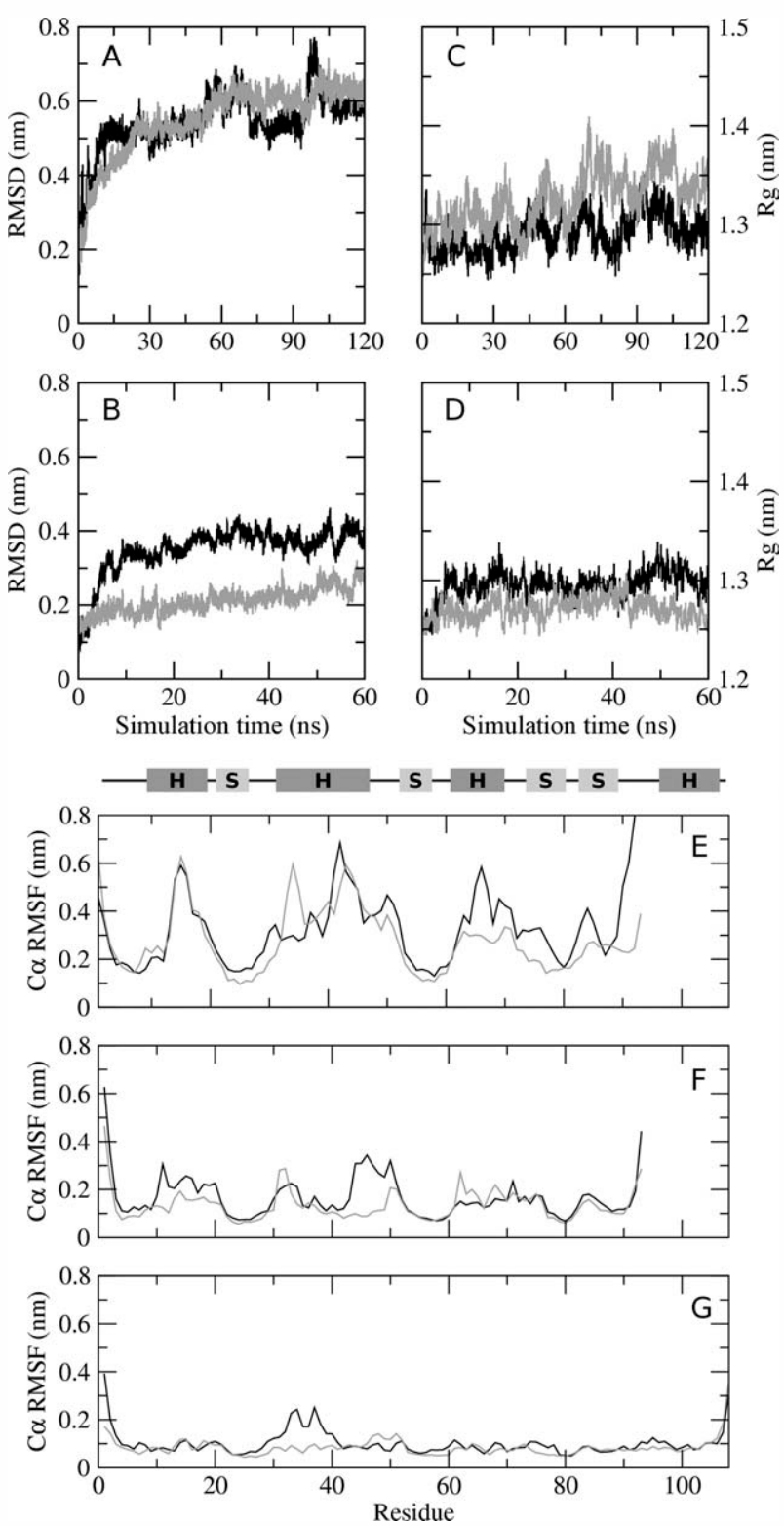

Figure 9

Molecular dynamics simulation of fragment TRX1-93 and the complex TRX1-93/TRX94-108. Root mean square deviations (RMSD) of the C $\alpha$ positions of the fragment TRX1-93 in the absence (A) or in the presence (B) of peptide TRX94-108 are plotted along the trajectory. In the right panels, the calculated radius of gyration $\left(R_{\mathrm{g}}\right)$ for the fragment TRX1-93 in the absence $(\mathbf{C})$ or in the presence (D) of peptide TRX94108 is shown. The root mean square fluctuation (RMSF) of the C $\alpha$ coordinates was calculated along the trajectory as indicated in Materials and Methods; fragment TRX1-93 in the absence (E) or in the presence (F) of peptide TRX94-108. Two MDS (in black and gray) were run for each simulation condition. The position of secondary structure elements along the TRX sequence is shown in grey boxes where $\mathrm{H}$ and $\mathrm{S}$ represent $\alpha$-helices and $\beta$-strands, respectively. (G) In addition, RMSF of the C $\alpha$ coordinates was also calculated along the MDS of the fulllength TRX. 

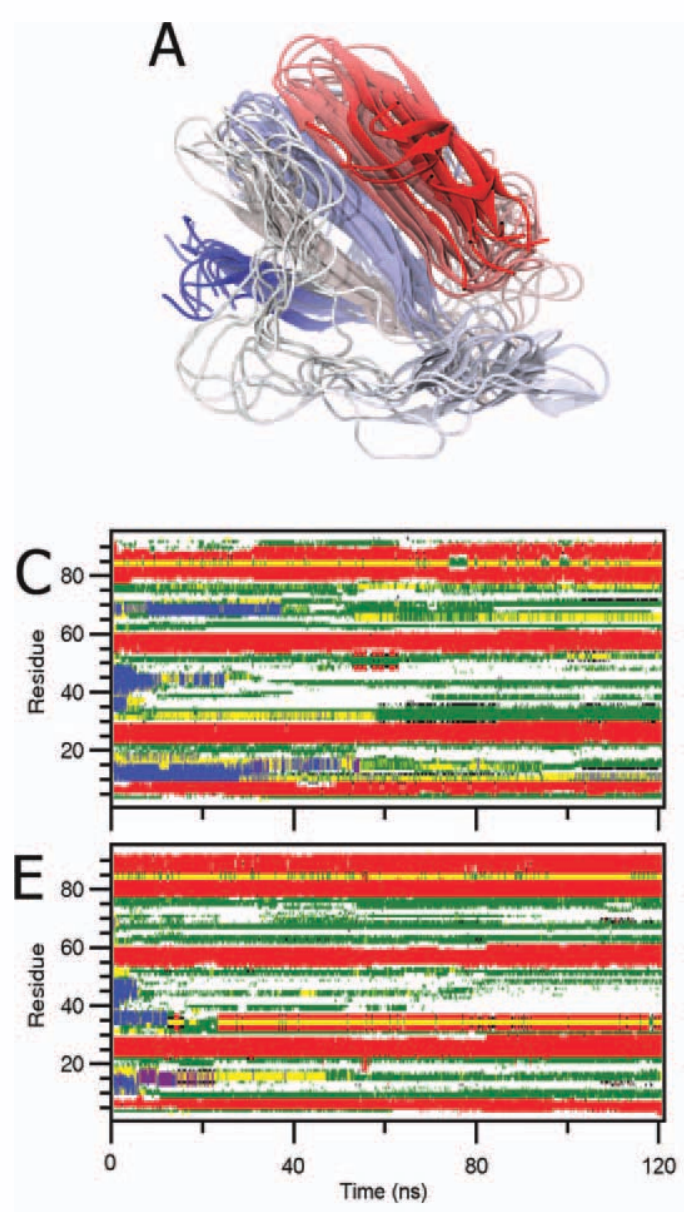

口 coil $\square$ B-She
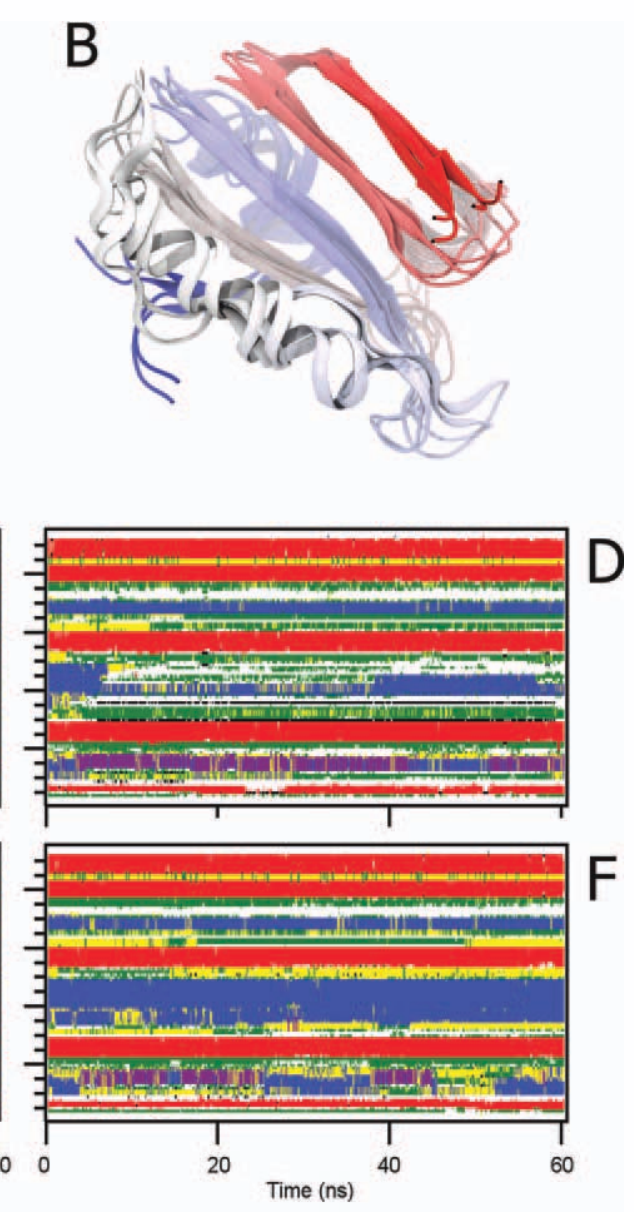

\section{Figure 10}

Representative structures of the conformational ensembles obtained after two independent simulations run for the fragment TRX1-93 in the absence (A) or in the presence (B) of peptide TRX94-108. The structures arise from a cluster analysis on a C $\alpha$ RMSD matrix, and they represent at least $90 \%$ of the total ensemble. Secondary structure propensities along the MDS were calculated for each residue with the program DSSP 75,76 and the different elements are indicated in color at the bottom. Two runs are plotted for each system, fragment TRX1-93 (C and E) and the complex TRX1-93/TRX94-108 (D and F).

that the native-like structure of the fragment becomes favored.

On the other hand, at high protein concentrations fragment TRX1-93 reversibly aggregates to yield a nonnative oligomer, as judged by near-UV CD, fluorescence, and DOSY-NMR spectroscopies. This multimeric ensemble likely establishes intermolecular contacts involving partially exposed apolar surfaces, as attested experimentally by ANS binding. ${ }^{47}$ Furthermore, MDS results herein included shed light on the probable origin of this process: in the absence of the cognate peptide TRX94-108, the amino acid stretch 35-50 (including $\alpha$-helices 2 and 3 in the native structure) stabilizes an extended conformation. Nevertheless, the fragment remains relatively compact, as judged by its behavior in SEC-FPLC run at low protein concentrations, ${ }^{47}$ a fact supported by MDS as well [Fig. 9(C,D)]. In addition, along these simulations the persistence of $\beta$ elements in the fragment might endow it with the ability of establishing quaternary interactions, thus providing support for the observation that this truncated form results prone to reversibly aggregate in the absence of the cognate peptide. By the same token, this might result in the observed slow refolding kinetics of the complex triggered by the addition of the peptide. Interestingly, the existence of a burst intermediate with higher content of $\beta$-structure than the native state was experimentally observed after reconstruction of the farUV CD spectrum along the first steps of the folding reaction of TRX, as measured by a combination of continuous and stopped-flow methods. ${ }^{78}$ In this scenario, we speculate that fragment TRX1-93 might share features of this on-pathway intermediate form.

When the non-native ensemble represented by fragment TRX1-93 is mixed with the peptide, it slowly 
evolves into a monomeric and compact structure. The low speed of this folding reaction, as attested by the appearance of the near-UV CD signature (Fig. 4), enabled us to follow it by real-time NMR techniques. The similar rate of development of ${ }^{1} \mathrm{H}-{ }^{15} \mathrm{~N}$ HSQC crosspeak intensities suggests that in the dominant phenomenon of native-like complex assembly, the dissociation of the oligomer is the rate-limiting step. Significantly, the spread of chemical shifts in the endpoint ${ }^{1} \mathrm{H}-{ }^{15} \mathrm{~N}$ HSQC experiment shows up as a spectrum perfectly superimposable to that rendered from an equilibrium experiment (Fig. 3). In due course, the latter keeps close resemblance to the spectrum of full-length TRX. ${ }^{49}$ Nevertheless, significant localized changes do occur and point to the amino acid stretch 23-42, a section of the polypeptide chain comprising the active site residues of TRX. Here, one should be reminded that the complex does recover catalytic activity, although to a lesser extent (7.1\%) than the full-length protein. ${ }^{47}$ Small environmental perturbations, as attested by chemical shifts differences for selected amide backbone resonances in this region, could reasonably account for this fact.

Importantly, the TRX complex not only adopts a remarkably similar secondary and tertiary structure, but also shares an overall backbone dynamics comparable to native full-length TRX. However, a more detailed picture, as revealed by NOE values at residue level slightly but consistently lower than those for full-length TRX, demonstrates an enhanced dynamics for the complex. In addition, the pattern of $R_{2}$ values would indicate a decrease of the slower motions (i.e., those in the ns regime) for residues belonging to helix $\alpha 3$ and to the $\beta$ hairpin at the C-terminus and/or, more likely, the occurrence of an exchange process involving the interacting surface with the peptide.

Remarkably, equilibrium titration experiments described here (Fig. 7) allow us to measure a $K_{\mathrm{D}}$ value for peptide TRX94-108/fragment TRX1-93 interaction compatible with measurements by ITC. ${ }^{47}$ In addition, NMR experiments enabled us to infer that localized structural alterations are indeed involved in the process of peptide binding, as attested by the significant chemical shift differences experienced by residues I4, F27, W31, C35, A39, I41, V91, G92, and A93. This evidence points to these residues undergoing fast conformational rearrangements, presumably due to the binding of a second peptide molecule to the surface of the complex. It is noteworthy to point out that all residues mentioned above share a distinct apolar character and some of them form part of the active site groove.

Interestingly, the solution NMR structure of human TRX, in complex with a target peptide from the transcription factor NF $\kappa B(\mathrm{PDB}$ ID $=1 \mathrm{MDI}),{ }^{79}$ shows that the interaction surface between both parts involvesamong other residues-S90, G91, and A92 from the Cterminal strand and residues of the active site including
W31. Moreover, the structure of Mycobacterium tuberculosis TRX C (MtbTrxC, PDB ID $=2 \mathrm{IIU})^{80}$ shows that the partially unfolded C-terminal $\alpha$-helix peptide interacts with the apolar groove of a MtbTrxC symmetry mate. Both examples point to the same region on the surface of TRX, which could be common with the putative second binding site for TRX94-108 on the surface of our TRX complex, as inferred from titration experiments. However, we cannot exclude that conformational rearrangements distant $(>10 \AA)$ from the active site region could also be taking place as a consequence of this second binding event. Proof of this is the peptide concentration dependent change in chemical shift detected for solvent inaccessible amino acid residues I4 and I41 (solvent accessible surface area $=8.9$ and $9.9 \%$, respectively).

To further illustrate the conformational plasticity inherent to TRX, a new crystal structure of human TRX (PDB $\mathrm{ID}=3 \mathrm{E} 3 \mathrm{E}$ ) reveals ample room for conformational changes in this fold: the unraveling of a helix (corresponding to helix $\alpha 4$ in E. coli TRX) triggers a more open conformation and an elongated hydrophobic pocket in its place. ${ }^{81}$ Taking into account crystal packing effects, this example proves the extreme ability of TRX to tolerate long range perturbations while maintaining its overall fold.

Interestingly, by applying MDS on site-specific mutants of the peptide, we observed that $\alpha$-helix 94-108 unwinds concertedly with $\alpha$-helix 3.49 Inspired by this previous evidence, the MDS results and the kinetics data presented herein enabled us to suggest that the reorganization of the stretch 35-50 into helical elements, occurring upon binding of peptide TRX 94-108 to fragment TRX1-93, might result critical for the consolidation of the overall TRX tertiary structure.

Altogether, structural and dynamic evidence presented in this paper points to the key role played by the C-terminal helical module in conferring reduced full-length TRX with the ability to act as a cooperative folding unit.

\section{ACKNOWLEDGMENTS}

The authors specially thank Lucia Chemes for assistance in CD experiments and kinetic analysis. The authors also acknowledge valuable comments on NMR data analysis by Mariana Gallo. The Bruker Avance II $600 \mathrm{MHz}$ NMR spectrometer used in this work was purchased from ANPCyT and CONICET.

\section{REFERENCES}

1. Daggett V, Fersht A. The present view of the mechanism of protein folding. Nat Rev Mol Cell Biol 2003;4:497-502.

2. Ferguson N, Fersht AR. Early events in protein folding. Curr Opin Struct Biol 2003;13:75-81.

3. Fersht AR. Nucleation mechanisms in protein folding. Curr Opin Struct Biol 1997;7:3-9.

4. Ferreiro DU, Hegler JA, Komives EA, Wolynes PG. Localizing frustration in native proteins and protein assemblies. Proc Natl Acad Sci USA 2007;104:19819-19824. 
5. Ferreiro DU, Hegler JA, Komives EA, Wolynes PG. On the role of frustration in the energy landscapes of allosteric proteins. Proc Natl Acad Sci USA 2011;108:3499-3503.

6. Onuchic JN, Luthey-Schulten Z, Wolynes PG. Theory of protein folding: the energy landscape perspective. Annu Rev Phys Chem 1997;48:545-600.

7. Onuchic JN, Wolynes PG. Theory of protein folding. Curr Opin Struct Biol 2004;14:70-75.

8. Haran G. How, when and why proteins collapse: the relation to folding. Curr Opin Struct Biol 2011;22:1-7.

9. Bucher D, Grant BJ, McCammon JA. Induced fit or conformational selection? The role of the semi-closed state in the maltose binding protein. Biochemistry 2011;50:10530-10539.

10. Chi CN, Bach A, Engstrom A, Wang H, Stromgaard K, Gianni S, Jemth P. A sequential binding mechanism in a PDZ domain. Biochemistry 2009;48:7089-7097.

11. Qin F, Jiang Y, Chen Y, Wu M, Yan G, Ye W, Li Y, Zhang J, Chen HF. Conformational selection or induced fit for Brinker and DNA recognition. Phys Chem Chem Phys 2011;13:1407-1412.

12. Scott DR, Borbulevych OY, Piepenbrink KH, Corcelli SA, Baker BM. Disparate degrees of hypervariable loop flexibility control Tcell receptor cross-reactivity, specificity, and binding mechanism. J Mol Biol 2011;414:385-400.

13. Tobi D, Bahar I. Structural changes involved in protein binding correlate with intrinsic motions of proteins in the unbound state. Proc Natl Acad Sci USA 2005;102:18908-18913.

14. Csermely P, Palotai R, Nussinov R. Induced fit, conformational selection and independent dynamic segments: an extended view of binding events. Trends Biochem Sci 2010;35:539-546.

15. Zhuravlev PI, Papoian GA. Protein functional landscapes, dynamics, allostery: a tortuous path towards a universal theoretical framework. Q Rev Biophys 2010;43:295-332.

16. Aleksandrov A, Simonson T. Molecular dynamics simulations show that conformational selection governs the binding preferences of imatinib for several tyrosine kinases. J Biol Chem 2010;285:1380713815.

17. Bhardwaj A, Cingolani G. Conformational selection in the recognition of the snurportin importin beta binding domain by importin beta. Biochemistry 2010;49:5042-5047.

18. Hammes GG, Chang YC, Oas TG. Conformational selection or induced fit: a flux description of reaction mechanism. Proc Natl Acad Sci USA 2009;106:13737-13741.

19. Hu J, Li D, Su XD, Jin C, Xia B. Solution structure and conformational heterogeneity of acylphosphatase from Bacillus subtilis. FEBS Lett 2010;584:2852-2856.

20. Rea AM, Thurston V, Searle MS. Mechanism of ligand-induced folding of a natively unfolded helixless variant of rabbit I-BABP. Biochemistry 2009;48:7556-7564.

21. Wlodarski T, Zagrovic B. Conformational selection and induced fit mechanism underlie specificity in noncovalent interactions with ubiquitin. Proc Natl Acad Sci USA 2009;106:19346-19351.

22. Zhou HX. From induced fit to conformational selection: a continuum of binding mechanism controlled by the timescale of conformational transitions. Biophys J 2010;98:15-17.

23. Grunberg R, Leckner J, Nilges M. Complementarity of structure ensembles in protein-protein binding. Structure 2004;12: 2125-2136.

24. Rose GD, Fleming PJ, Banavar JR, Maritan A. A backbone-based theory of protein folding. Proc Natl Acad Sci USA 2006;103:1662316633.

25. Hilser VJ, Garcia-Moreno EB, Oas TG, Kapp G, Whitten ST. A statistical thermodynamic model of the protein ensemble. Chem Rev 2006;106:1545-1558.

26. Schrank TP, Bolen DW, Hilser VJ. Rational modulation of conformational fluctuations in adenylate kinase reveals a local unfolding mechanism for allostery and functional adaptation in proteins. Proc Natl Acad Sci USA 2009;106:16984-16989.
27. Lange OF, Lakomek NA, Fares C, Schroder GF, Walter KF, Becker S, Meiler J, Grubmuller H, Griesinger C, de Groot BL. Recognition dynamics up to microseconds revealed from an RDC-derived ubiquitin ensemble in solution. Science 2008;320:1471-1475.

28. Bolen DW, Rose GD. Structure and energetics of the hydrogenbonded backbone in protein folding. Annu Rev Biochem 2008;77:339-362.

29. Hu CY, Pettitt BM, Roesgen J. Osmolyte solutions and protein folding. F1000 Biol Rep 2009;1:41.

30. Russo AT, Rosgen J, Bolen DW. Osmolyte effects on kinetics of FKBP12 C22A folding coupled with prolyl isomerization. J Mol Biol 2003;330:851-866.

31. Wilke CO, Drummond DA. Signatures of protein biophysics in coding sequence evolution. Curr Opin Struct Biol 2010;20: 385-389.

32. Sanchez IE, Tejero J, Gomez-Moreno C, Medina M, Serrano L. Point mutations in protein globular domains: contributions from function, stability and misfolding. J Mol Biol 2006;363:422-432.

33. Godoy-Ruiz R, Ariza F, Rodriguez-Larrea D, Perez-Jimenez R, Ibarra-Molero B, Sanchez-Ruiz JM. Natural selection for kinetic stability is a likely origin of correlations between mutational effects on protein energetics and frequencies of amino acid occurrences in sequence alignments. J Mol Biol 2006;362:966-978.

34. Smock RG, Gierasch LM. Sending signals dynamically. Science 2009;324:198-203.

35. Tokuriki N, Tawfik DS. Protein dynamism and evolvability. Science 2009;324:203-207.

36. Lee GM, Craik CS. Trapping moving targets with small molecules. Science 2009;324:213-215.

37. Collet JF. A new protein system protects single cysteines against oxidative stress. [Article in French] Bull Mem Acad R Med Belg 2010;165:299-305.

38. Dyson HJ, Gippert GP, Case DA, Holmgren A, Wright PE. Threedimensional solution structure of the reduced form of Escherichia coli thioredoxin determined by nuclear magnetic resonance spectroscopy. Biochemistry 1990;29:4129-4136.

39. Katti SK, LeMaster DM, Eklund H. Crystal structure of thioredoxin from Escherichia coli at 1.68 A resolution. J Mol Biol 1990;212:167184.

40. Chandrasekhar K, Krause G, Holmgren A, Dyson HJ. Assignment of the 15N NMR spectra of reduced and oxidized Escherichia coli thioredoxin. FEBS Lett 1991;284:178-183.

41. Stone MJ, Chandrasekhar K, Holmgren A, Wright PE, Dyson HJ. Comparison of backbone and tryptophan side-chain dynamics of reduced and oxidized Escherichia coli thioredoxin using 15N NMR relaxation measurements. Biochemistry 1993;32:426-435.

42. Jeng MF, Dyson HJ. Comparison of the hydrogen-exchange behavior of reduced and oxidized Escherichia coli thioredoxin. Biochemistry 1995;34:611-619.

43. Yang XM, Georgescu RE, Li JH, Yu WF, Haierhan, Tasayco ML. Recognition between disordered polypeptide chains from cleavage of an alpha/beta domain: self-versus non-self-association. Pac Symp Biocomput 1999;590-600.

44. Slaby I, Holmgren A. Structure and enzymatic functions of thioredoxin refolded by complementation of two tryptic peptide fragments. Biochemistry 1979;18:5584-5591.

45. Qin J, Clore GM, Gronenborn AM. The high-resolution threedimensional solution structures of the oxidized and reduced states of human thioredoxin. Structure 1994;2:503-522.

46. Louis JM, Georgescu RE, Tasayco ML, Tcherkasskaya O, Gronenborn AM. Probing the structure and stability of a hybrid protein: the human-E. coli thioredoxin chimera. Biochemistry 2001;40: $11184-11192$.

47. Santos J, Marino-Buslje C, Kleinman C, Ermacora MR, Delfino JM. Consolidation of the thioredoxin fold by peptide recognition: interaction between E. coli thioredoxin fragments 1-93 and 94-108. Biochemistry 2007;46:5148-5159. 
48. Roman EA, Rosi P, Lebrero MC, Wuilloud R, Flecha FL, Delfino JM, Santos J. Gain of local structure in an amphipathic peptide does not require a specific tertiary framework. Proteins 2010;78:2757-2768.

49. Santos J, Sica MP, Buslje CM, Garrote AM, Ermacora MR, Delfino JM. Structural selection of a native fold by peptide recognition. Insights into the thioredoxin folding mechanism. Biochemistry 2009;48:595-607.

50. Schagger H, von Jagow G. Tricine-sodium dodecyl sulfate-polyacrylamide gel electrophoresis for the separation of proteins in the range from 1 to $100 \mathrm{kDa}$. Anal Biochem 1987;166:368-379.

51. Berjanskii MV, Wishart DS. A simple method to predict protein flexibility using secondary chemical shifts. J Am Chem Soc 2005;127:14970-14971.

52. Berjanskii M, Wishart DS. NMR: prediction of protein flexibility. Nat Protoc 2006;1:683-688.

53. Marsh JA, Singh VK, Jia Z, Forman-Kay JD. Sensitivity of secondary structure propensities to sequence differences between alphaand gamma-synuclein: implications for fibrillation. Protein Sci 2006;15:2795-2804.

54. Serrano L. Comparison between the phi distribution of the amino acids in the protein database and NMR data indicates that amino acids have various phi propensities in the random coil conformation. J Mol Biol 1995;254:322-333.

55. West $\mathrm{Nj}$, Smith LJ. Side-chains in native and random coil protein conformations. Analysis of NMR coupling constants and chil torsion angle preferences. J Mol Biol 1998;280:867-877.

56. Schanda P, Brutscher B. Very fast two-dimensional NMR spectroscopy for real-time investigation of dynamic events in proteins on the time scale of seconds. J Am Chem Soc 2005;127:8014-8015.

57. Wilkins DK, Grimshaw SB, Receveur V, Dobson CM, Jones JA, Smith LJ. Hydrodynamic radii of native and denatured proteins measured by pulse field gradient NMR techniques. Biochemistry 1999;38:16424-16431.

58. Schanda P, Forge V, Brutscher B. HET-SOFAST NMR for fast detection of structural compactness and heterogeneity along polypeptide chains. Magn Reson Chem 2006;44:S177-S184.

59. Farrow NA, Zhang O, Forman-Kay JD, Kay LE. A heteronuclear correlation experiment for simultaneous determination of $15 \mathrm{~N}$ longitudinal decay and chemical exchange rates of systems in slow equilibrium. J Biomol NMR 1994;4:727-734.

60. Farrow NA, Zhang O, Forman-Kay JD, Kay LE. Comparison of the backbone dynamics of a folded and an unfolded $\mathrm{SH} 3$ domain existing in equilibrium in aqueous buffer. Biochemistry 1995;34:868-878.

61. Farrow NA, Zhang O, Forman-Kay JD, Kay LE. Characterization of the backbone dynamics of folded and denatured states of an SH3 domain. Biochemistry 1997;36:2390-2402.

62. Oostenbrink C, Villa A, Mark AE, van Gunsteren WF. A biomolecular force field based on the free enthalpy of hydration and solvation: the GROMOS force-field parameter sets 53A5 and 53A6. J Comput Chem 2004;25:1656-1676.

63. Van Der Spoel D, Lindahl E, Hess B, Groenhof G, Mark AE, Berendsen HJ. GROMACS: fast, flexible, and free. J Comput Chem 2005;26:1701-1718.

64. Berendsen HJC, Grigera JR, Straatsma TP. The missing term in effective pair potentials. J Phys Chem 1987;91:6269-6271.
65. Berendsen HJC, Postma JPM, Gunsteren WFv, DiNola A, Haak JR. Molecular dynamics with coupling to an external bath. J Chem Phys 1984;81:3684-3690.

66. Berendsen HJC, van Gunsteren WF. Molecular dynamics simulations: techniques and approaches. In: Orville-Thomas WJ, Barnes AJ, Yarwood J, editors. Molecular liquids-dynamics and interactions. Proceedings of NATO ASI, Reidel, Dordrecht, 1984. pp 475500 .

67. Hess B, Bekker H, Berendsen HJC, Fraaije JGEM. LINCS: a linear constraint solver for molecular simulations. J Comput Chem 1997;18:1463-1472.

68. Feenstra KA, Hess B, Berendsen HJC. Improving efficiency of large time-scale molecular dynamics simulations of hydrogen-rich systems. J Comput Chem 1999;20:786-798.

69. Tironi IG, Sperb R, Smith PE, Gunsteren WFv. A generalized reaction field method for molecular dynamics simulations. J Chem Phys 1995;102:5451-5459.

70. Daura X, Gademann K, Jaun B, Seebach D, van Gunsteren WF, Mark AE. Peptide folding: when simulation meets experiment. Angew Chem Int Ed 1999;38:236-240.

71. Garcia De La Torre J, Huertas ML, Carrasco B. Calculation of hydrodynamic properties of globular proteins from their atomiclevel structure. Biophys J 2000;78:719-730.

72. Wishart DS, Sykes BD. The 13C chemical-shift index: a simple method for the identification of protein secondary structure using 13C chemical-shift data. J Biomol NMR 1994;4:171-180.

73. Wishart DS, Sykes BD. Chemical shifts as a tool for structure determination. Methods Enzymol 1994;239:363-392.

74. Humphrey W, Dalke A, Schulten K. VMD: visual molecular dynamics. J Mol Graph 1996;14:33-38,27-28.

75. Kabsch W, Sander C. How good are predictions of protein secondary structure? FEBS Lett 1983;155:179-182.

76. Kabsch W, Sander C. Dictionary of protein secondary structure: pattern recognition of hydrogen-bonded and geometrical features. Biopolymers 1983;22:2577-2637.

77. Langsetmo K, Fuchs J, Woodward C. Escherichia coli thioredoxin folds into two compact forms of different stability to urea denaturation. Biochemistry 1989;28:3211-3220.

78. Georgescu RE, Li JH, Goldberg ME, Tasayco ML, Chaffotte AF. Proline isomerization-independent accumulation of an early intermediate and heterogeneity of the folding pathways of a mixed alpha/beta protein, Escherichia coli thioredoxin. Biochemistry 1998;37:10286-10297.

79. Qin J, Clore GM, Kennedy WM, Huth JR, Gronenborn AM. Solution structure of human thioredoxin in a mixed disulfide intermediate complex with its target peptide from the transcription factor NF kappa B. Structure 1995;3:289-297.

80. Hall G, Shah M, McEwan PA, Laughton C, Stevens M, Westwell A, Emsley J. Structure of mycobacterium tuberculosis thioredoxin C. Acta Crystallogr D Biol Crystallogr 2006;62:1453-1457.

81. Hall G, Emsley J. Structure of human thioredoxin exhibits a large conformational change. Protein Sci 2010;19:1807-1811. 